\title{
Moving Block Sequence and Organizational Evolutionary \\ Algorithm for General Floorplanning with Arbitrarily Shaped Rectilinear Blocks
}

\author{
Jing Liu ${ }^{1}$, Member, IEEE $\quad$ Weicai Zhong ${ }^{1}$, Member, IEEE $\quad$ Licheng Jiao ${ }^{1}$, Senior Member, IEEE, \\ Xue $\mathrm{Li}^{2}$, Member IEEE \\ ${ }^{1}$ Institute of Intelligent Information Processing, Xidian University, Xi'an 710071， China \\ ${ }^{2}$ School of Information Technology and Electrical Engineering, The University of Queensland, Brisbane, \\ Qld 4072, Australia \\ E-mail: neouma@163.com
}

Abstract—A new nonslicing floorplan representation, the moving block sequence (MBS), is proposed in this paper. Our idea of the MBS originates from the observation that placing blocks on a chip has some similarities to playing the game, Tetris ${ }^{\circledR}$. Because no extra constraints are exerted on solution spaces, the MBS is not only useful for evolutionary algorithms, but also for dealing with rectangular, convex rectilinear, and concave rectilinear blocks, similarly and simultaneously, without partitioning rectilinear blocks into sub-blocks. This is owed to a special structure designed for recording the information of both convex and concave rectilinear blocks in a uniform form. Theoretical analyses show that the computational cost of transforming an MBS to a floorplan with rectangular blocks, in terms of the number of blocks, is between linear and quadratic. Furthermore, as a follow-up of our previous works, a new organizational evolutionary algorithm (OEA) based on the MBS (MBS-OEA) is proposed. With the intrinsic properties of the MBS in mind, three new evolutionary operators are designed in the MBS-OEA. To test the performance of the MBS-OEA, benchmarks with hard rectangular, soft rectangular, and hard rectilinear blocks are used. The number of blocks in these benchmarks varies from 9 to 
300. Also, the MBS-OEA and several well-designed existing algorithms are compared. The results show that the MBS-OEA can find high quality solutions for various problems. Additionally, the MBS-OEA shows a good performance in solving the problems with 300 hard rectangular blocks, 100 soft rectangular blocks, and 100 hybrid blocks, including both soft rectangular and hard rectilinear blocks. This illustrates that the MBS-OEA is not only suitable for solving a wide range of problems, but also competent for solving large-scale problems. Finally, a set of specific experiments is designed to identify the key component that is mainly responsible for the good performance of the MBS-OEA.

Index Terms — Very large scale integration, Floorplanning, Moving block sequence, Evolutionary algorithms, Organization 


\section{Introduction}

Floorplanning is an essential step in the hierarchical physical design of deep sub-micron very large scale integration (VLSI) circuits. It involves planning the positions and shapes of a set of blocks on a chip to optimize circuit performances. Blocks must be placed without being overlapped. As the technology moves into the deep sub-micron era, circuit sizes and complexities are growing rapidly and floorplanning has become even more important than it was before. Although floorplanning has been studied extensively, most previous works used simulated annealing (SA) as the search algorithm and handled rectilinear blocks by partitioning them into a set of rectangular sub-blocks. Therefore, it is still challenging and practically useful to find a nonslicing floorplan representation that is not only suitable for evolutionary algorithms (EAs) because of their high potential, but can also handle both rectangular and rectilinear blocks, similarly and simultaneously, without partitioning rectilinear blocks into sub-blocks. This paper presents the moving block sequence (MBS) as a new nonslicing floorplan representation to meet the above requirements.

Our idea of the MBS originates from the observation that placing blocks on a chip has some similarities to playing the game, Tetris ${ }^{\circledR}$. As in Tetris ${ }^{\circledR}$, we place blocks on a chip, one by one. Each block starts from an initial position and moves on the chip, until it reaches an appropriate position. In Tetris ${ }^{\circledR}$, each block always appears on the top of the screen, and it is the players who find an appropriate position for each block during the descending process. In the MBS, on the other hand, four initial positions are designed from where the blocks could be moved to other places, according to the move rules.

An MBS is designed to be composed of two tuples, one denoting the permutation of 
block names and the other the initial positions of blocks. Each block is placed on the chip in the order it occurs in the permutation. There are four choices for the initial position and no extra constraints are exerted on the solution space. Hence, it is easy to design effective crossover operators for EAs on such a solution space. Owing to a special structure designed for recording the information of both convex and concave rectilinear blocks, the MBS can handle them in the same way as the rectangular blocks, namely, without partitioning them into sub-blocks. The MBS has a smaller solution space, $\left(n ! 2^{2(n-1)}\right)$, than several existing representations, such as the sequence pair (SP) [1], the bounded-sliceline grid (BSG) [2], the transitive closure graph (TCG) [3], the TCG-S [4], the corner sequence (CS) [5], and so on. The solution space of each of the SP, the TCG, the TCG-S, and the CS is $(n !)^{2}$, and that of the BSG is $n ! C\left(n^{2}, n\right)$, where $n$ is the number of blocks.

From another viewpoint, the MBS can also be considered an extension of the bottom-left (BL) method, the most documented heuristic approach in the field of cutting and packing, because the BL has only one initial position. Other similarities and differences between the MBS and the BL are detailed in Subsection II.C.

On the basis of the MBS, a new organizational evolutionary algorithm (OEA), the MBS-OEA, is proposed. OEAs are a new kind of EA proposed in our previous works, and have been successfully applied to classification problems [6], numerical optimization problems [7], and satisfiability problems [8]. In this paper, three new operators, namely, the splitting operator, the annexing operator, and the training operator are designed on the basis of the MBS, so that the framework of the OEA is applicable to solving floorplanning problems.

This paper is organized as follows. Section II discusses some related work. Section III 
gives the definition of the moving block sequence. Section IV presents the algorithm transforming an MBS to a floorplan. Section V introduces the MBS-OEA in detail. Experiments are given in Section VI. Section VII provides an experimental study that aims to identify the mechanism that is mainly responsible for the effectiveness of the MBS-OEA. Finally, some conclusions and future work are provided in Section VIII.

\section{Related Work}

Many researchers proposed various algorithms for floorplanning problems by applying different mathematical tools. Among these, stochastic optimization algorithms were the most popular and had attracted increasing attention. These algorithms employed methods of perturbing floorplans and searching for better solutions. As this kind of method can design specific operations based on the characteristics and complexities of problems, the quality of solutions was generally high. To optimize floorplans by stochastic optimization algorithms, the representation is one of the key and fundamental issues.

The representation has been studied extensively. In general, there are two kinds of floorplans, slicing and nonslicing. A slicing floorplan can be obtained by recursively cutting rectangles horizontally or vertically into smaller rectangles; otherwise, it is a nonslicing floorplan. Slicing floorplan representations have smaller solution spaces and can describe any slicing structure with no redundancy. But in practical applications, most designs belong to nonslicing floorplans.

Therefore, nonslicing floorplan representations have recently attracted much attention. Although this kind of representation is of more general utility and can utilize the area more effectively and achieve better routability, it is more complex and difficult to embody such 
representations. There were no such efficient representations other than the constraint graphs until the SP [1] and the BSG [2] appeared in the mid-1990s. Subsequently, several efficient representations were proposed, such as the $O$-tree [9], the $B^{*}$-tree [10], the corner block list (CBL) [11], the TCG [3], the TCG-S [4], the twin binary sequences (TBS) [12], the CS [5], and so on. However, most stochastic optimization algorithms using these representations were SA. Moreover, these representations were designed for rectangular blocks and cannot handle rectilinear blocks directly.

\section{A. Stochastic Optimization Algorithms}

In the field of floorplanning, SA is much more popular than EAs. Several researchers used general SA to search solution spaces [1]-[5], [10]-[12]. They perturbed the current floorplan according to properties of the employed representation and decreased the temperature according to a pre-defined cooling schedule. Additionally, on the basis of the SP, [13] proposed an orthogonal SA algorithm (OSA) with an efficient generation mechanism (EGM) for solving floorplanning problems. The EGM sampled a small number of representative floorplans and then efficiently derived a high-performance floorplan using a systematic reasoning method for the next move of the OSA, based on the orthogonal experimental design.

In recent years, EAs have attracted increasing attention because they are suitable for solving complex and ill-defined problems. They have been successfully applied to the fields of numerical optimization [14], constraint satisfaction problems [15], data mining and knowledge discovery [16], [17], neural networks [18], and so on.

There were also a few studies on the application of EAs to floorplanning problems. 
Cohoon et al. in [19] offered the classical work on using genetic algorithms (GAs) for floorplanning. The arrangement of rooms on the layout surface was represented in the genotype by a postfix expression that was not normalized of the corresponding slicing tree. Schnecke et al. in [20] used a GA to manipulate the binary slicing tree directly for floorplanning. However, the crossover involved complex repair mechanisms simply to ensure that the offspring represented a legal slicing floorplan. Valenzuela et al. in [21] presented a GA that used a slicing tree construction process. They used an order-based representation that encoded rectangles and binary operations into a simple permutation of structures and a decoder that converted the permutation of structures into a normalized postfix expression.

Generally speaking, applications of EAs to floorplanning problems are much fewer than those of SA. Although some researchers [19]-[21] used EAs, their works were all based on the slicing structure instead of the nonslicing structure. This restricted the popularization of these methods to some extent. In our opinion, the reason for fewer applications of EAs is that the nonslicing floorplan representations are generally very complex and solution spaces are constrained. In this case, EAs with traditional evolutionary operators (e.g., crossover) tend to create infeasible individuals during the search. To boost the development of EAs in the field of floorplanning, it is important to design new nonslicing floorplan representations that do not exert extra constraints on solution spaces and facilitate effective crossover operators.

\section{B. Floorplanning Problems with Arbitrarily Shaped Rectilinear Blocks}

In the simplest situation of floorplanning, all blocks are rectangular. However, in real design, as some of the circuit blocks come from design re-use, they are not necessarily rectangular. To fully optimize some predefined cost metric, for example, area, wirelength, or 
routability, it is important to deal with floorplanning problems with arbitrarily shaped rectilinear blocks. There are a few existing partition-based approaches that use the well-known representations.

Xu et al. in [22] explored the conditions of the feasible SP for L-shaped blocks. Kang et al. in [23] derived three necessary and sufficient conditions for recovering the shapes of convex rectilinear blocks based on the SP. Fujiyoshi et al. in [24] derived a necessary and sufficient condition of the feasible SP for rectilinear blocks. Nakatake et al. in [25] handled pre-placed and rectilinear blocks using the BSG. Kang et al. in [26] used the BSG and the SP to solve the topology constrained block packing for ordered convex rectilinear blocks and extended the method to handle arbitrary rectilinear blocks. Pang et al. in [27] and Wu et al. in [28] used the $O$-tree and the $B^{*}$-tree, respectively, to handle rectilinear blocks. Ma et al. in [29] used the CBL to deal with the placement abutment constraint and extended the method to deal with L- and T-shaped blocks. Lin et al. in [30] derived necessary and sufficient conditions of the feasible TCG for the sub-blocks. Additionally, Chu et al. in [31] dealt with the problem of giving a preliminary floorplan and changed the shapes and dimensions of some soft blocks to L-shaped to fill up the empty space.

From the literature cited, we can see that most previous works on floorplanning problems with rectilinear blocks partitioned rectilinear blocks into a set of rectangular sub-blocks and operated on sub-blocks under some constraints induced from the original rectilinear blocks. However, as the original rectilinear blocks were partitioned, some additional operations became necessary to retrieve the original shapes after packing, resulting in a larger computational cost. Thus, it is necessary to design a representation that can handle rectilinear 
blocks directly.

\section{Cutting and Packing Problems}

One might argue that the MBS is similar to the bottom-left (BL) and the bottom-left-fill (BLF) methods [32], [33] used in the field of cutting and packing. In the BL, each block starts from the top-right corner and slides as far as possible to the bottom, and then as far as possible to the left. These successive vertical and horizontal movements are repeated until blocks lock in a stable position. The BLF is a modified version of the BL and can fill the holes in placed rectangles.

Although all the BL, the BLF, and the MBS move blocks on a region, the $\mathrm{BL}$ and the BLF have only one initial position, namely, the top-right location, whereas the MBS has four initial positions. Moreover, the MBS has four move rules corresponding to the four initial positions. To illustrate the usefulness of these extra choices for the initial positions and the move rules in the MBS, a group of experiments on the MBS with only one or two initial positions is carried out and the results are presented in Subsection VII.A.

On the other hand, there is a major difference between cutting and packing problems and floorplanning. In the former, the width of the region is fixed and only the height needs to be minimized, whereas in the latter, there are no constraints on the regions, and the area needs to be minimized. Additionally, in the field of cutting and packing, problems are usually categorized into orthogonal problems (where blocks are rectangular) [34] and irregular problems [35]. The emphasis of the MBS is to handle rectilinear blocks, which are more general than rectangular blocks, but are a special case of irregular blocks.

EAs are also used in the field of cutting and packing. In 1985, Smith [36] first applied 
GAs to packing problems. At the same time, Davis [37] summarized the techniques for applications of GAs to epistatic domains using the example of two-dimensional (2D) bin packing. Since then, various packing problems, ranging from regular to arbitrary shapes in two or more dimensions, have been approached. In these approaches, individuals were represented as permutations of blocks. A placement heuristic was used to decode the representation by packing the pieces in the order given by the permutation. This approach remained popular for cutting and packing problems [38], [39]. There were also several approaches by incorporating the bound information of the branches of search trees into GAs [40]. Hopper and Turton [41] had reviewed applications of GAs to packing problems. They also conducted an empirical study of meta-heuristic and heuristic algorithms for 2D packing problems [42].

In the field of cutting and packing, because individuals are usually represented as permutations of blocks, the only task of EAs is to optimize the permutations. However, the MBS has two tuples, permutation and initial position. Thus, the task of the MBS-OEA is to optimize not only the permutation, but also the initial positions.

\section{Moving Block Sequence Representation}

The blocks in floorplanning can be classified into three types: hard rectangular blocks (HRaBs), soft rectangular blocks (SRaBs), and hard rectilinear blocks (HRiBs). Regardless of the type of blocks, the goal of floorplanning is to determine the shapes and locations of a set of blocks so that no block overlaps and to minimize some predefined cost metric induced by a floorplan. Each block is free to rotate.

Given a floorplanning problem with $n$ blocks, $\mathcal{B}=\left\{\boldsymbol{B}_{0}, \boldsymbol{B}_{1}, \ldots, \boldsymbol{B}_{n-1}\right\}$, let $\mathcal{F}(\mathcal{B})$ denote a 
floorplan and $\operatorname{Cost}(\mathcal{F}(\mathcal{B}))$ be the predefined cost metric. The cost metric can be the area of the minimum bounding rectangle (MBR) of $\mathcal{F}(\mathcal{B})$, the wirelength, or a linear combination of the area and the wirelength. Area minimization is one of the key objectives. Because the purpose of this paper is a "proof of concept", we limit the Cost function to the area. Other elements will be included in the cost function in future studies.

The shape and orientation of each block are fixed before applying the MBS. Because our idea of the MBS originates from the observation that a floorplan can be obtained by moving blocks on the chip according to some rules, designing rules for moves becomes the key task. In the following text, the bottom and left sides of the chip are fixed to form the $\mathrm{X}$ - and $\mathrm{Y}$-axis, respectively. Each block is first placed on an initial position from where it can move to other places at the first quadrant. The initial positions are defined as follows:

Definition 1: Let $\left(x_{\boldsymbol{B}}^{L B}, y_{\boldsymbol{B}}^{L B}\right)$, width $\boldsymbol{B}_{\boldsymbol{B}}$, and height $\boldsymbol{B}_{\boldsymbol{B}}$ denote the coordinate of the left-bottom corner, the width, and the height of the MBR of $\boldsymbol{B}$, respectively. Let $\boldsymbol{B o x}$ denote the MBR of the blocks that have been placed and $\left(x^{B o x R T}, y^{B o x R T}\right)$ denote the coordinate of the right-top corner of Box. The coordinate of the left-bottom corner of $\boldsymbol{B o x}$ is always (0, 0). Thus, there are four initial positions for $\boldsymbol{B}$, namely,

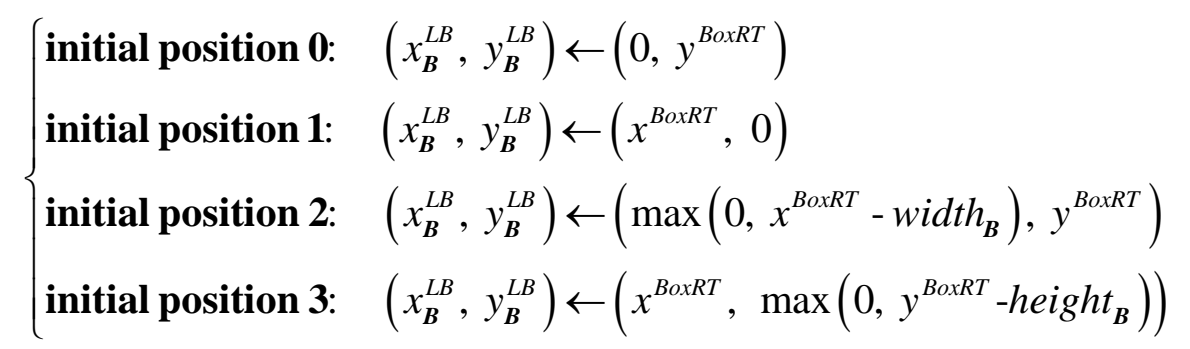

where $\max (a, b)$ denotes the larger one between $a$ and $b$.

According to Definition 1, the four initial positions for a new block are determined by the MBR of the blocks that have been placed and are illustrated in Fig.1(a). There are two special 
cases in initial positions 2 and 3, that is, the width or the height of $\boldsymbol{B}$ are larger than those of $\boldsymbol{B}$ ox. Thus, $\boldsymbol{B}$ must first slide rightward or upward until $x_{\boldsymbol{B}}^{L B}$ or $y_{\boldsymbol{B}}^{L B}$ is 0 , as shown in Figs.1(b) and (c).

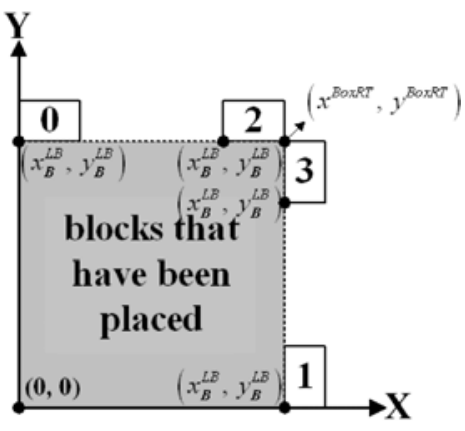

(a)

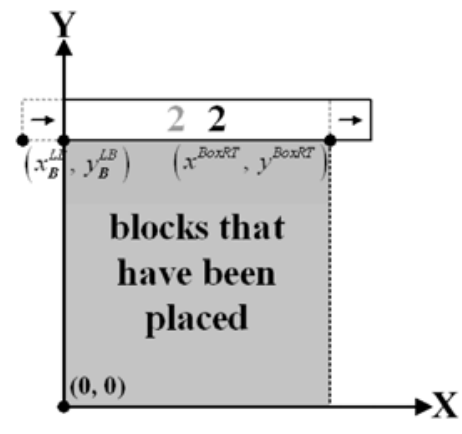

(b)

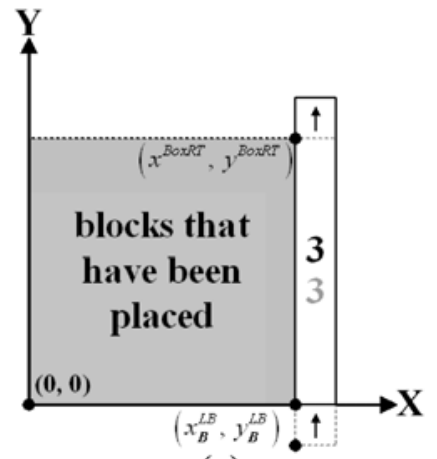

(c)

Fig.1. Initial positions: (a) 4 initial positions (b) the width of the block is larger than that of Box (c) the height of the block is larger than that of $\boldsymbol{B o x}$

Because we can fix the bottom and left edges of any floorplan and perform horizontal and vertical compaction iteratively, the final floorplan, after all compact operations converge, is an LB-compact floorplan with respect to the original one. Since the overall area of the LB-compact floorplan is less than or equal to that of the original one [9], the move rules are designed so that each block can move until an LB-compact floorplan is obtained.

Definition 2: There are four move rules corresponding to the four initial positions.

Move rule 0: When a block is put on initial position 0, this block can only slide downward until no downward movement is possible.

Move rule 1: When a block is put on initial position 1, this block can only slide leftward until no leftward movement is possible.

Move rule 2: When a block is put on initial position 2, this block can repeatedly slide downward and leftward, giving priority to downward movement so that this block only slides leftward if no downward movement is possible. 
Move rule 3: When a block is put on initial position 3, this block can repeatedly slide leftward and downward, giving priority to leftward movement so that this block only slides downward if no leftward movement is possible.

Definition 3: A Moving Block Sequence (MBS) has two tuples. One denotes the permutation of all block names, $\pi$, and the other denotes the initial positions (IPs) of all blocks, IP, namely,

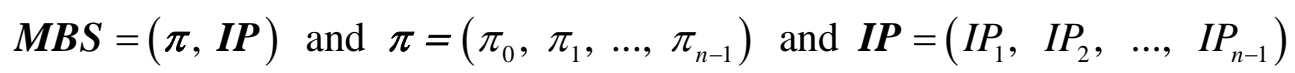

where $\pi_{i}(0 \leq i \leq n-1)$ denotes a block among $\left\{\boldsymbol{B}_{0}, \boldsymbol{B}_{1}, \ldots, \boldsymbol{B}_{n-1}\right\}$, and $I P_{j} \in\{0,1,2,3\}(1 \leq j \leq n-1)$ denotes the initial position for $\pi_{\mathrm{j}}$. $\pi_{0}$ is placed at the left-bottom corner of the first quadrant and $\pi_{1}, \pi_{2}, \ldots, \pi_{\mathrm{n}-1}$ are placed at the first quadrant in the order they occur in $\pi$.

As can be seen, there are always four choices for initial positions of any type of blocks and no extra constraints are exerted on solution spaces. Such a kind of solution space is suitable for designing effective crossover operators. Thus, the MBS is useful for EAs in solving floorplanning problems.

Theorem 1: Let $\mathcal{M B S}$ be the set of all possible MBSs for $n$ blocks; then, the size of the solution space $|\mathcal{M B S}|$ is equal to $\left(n ! 2^{2(n-1)}\right)$.

Proof: The number of combinations of $\pi$ is $n$ ! and that of $\boldsymbol{I P}$ is $\left(4^{(n-1)}\right)$. Thus,

$$
|\mathcal{M B S}|=n ! \times 4^{(n-1)}=n ! \times 2^{2(n-1)}
$$

\section{Algorithm Transforming an MBS to a Floorplan}

Although rectilinear blocks can be classified into two types, convex rectilinear blocks and concave rectilinear blocks, both boundaries are composed of a set of vertical and horizontal edges, and the distribution of these edges on the four orientations (top, bottom, left, and right) 
varies significantly. Thus, it is important to design a suitable structure to record the positions of these edges.

\section{A. Information Structure for Rectilinear Blocks}

All edges of a rectilinear block are parallel to the $\mathrm{X}$ - or the Y-axis. As the main operation of the MBS is moving a block leftward or downward, the problem of finding the left-most or the bottom-most position of a block is changed to that of judging the relative position between two edges, which are parallel either to the Y-axis or to the X-axis. Two structures, $\boldsymbol{e}^{/ / \mathrm{X}}$ and $\boldsymbol{e}^{/ / \mathrm{Y}}$, are designed to record the information of such edges.

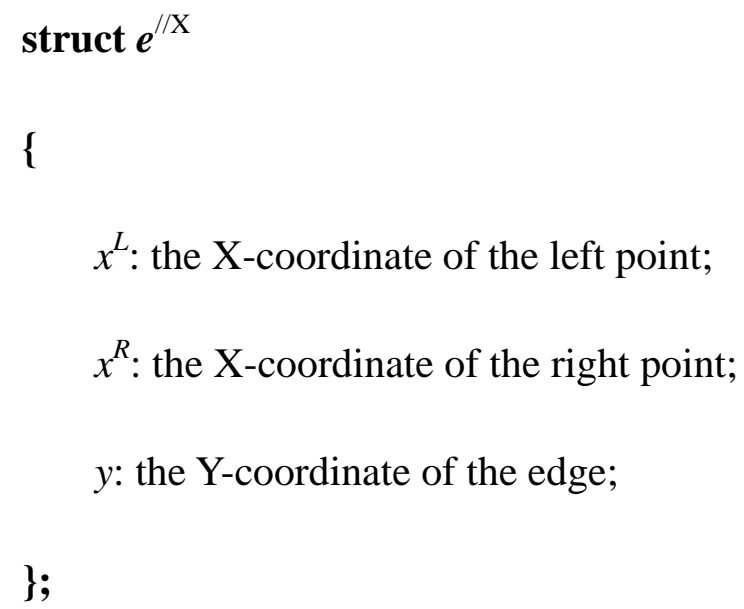

$x$ : the X-coordinate of the edge;

$y^{B}$ : the Y-coordinate of the bottom point;

$y^{T}$ : the Y-coordinate of the top point;

In the following text, $\boldsymbol{e}^{/ / \mathrm{X}}(\cdot)$ and $\boldsymbol{e}^{/ / \mathrm{Y}}(\cdot)$ denote the corresponding item in the above structures. 
The edges of a rectilinear block can be classified into four types.

Definition 4: Suppose sliding along the boundary of a rectilinear block clockwise. If the edge slides from right to left, it is a bottom edge; if it slides from left to right, it is a top edge; if it slides from bottom to top, it is a left edge; and if it slides from top to bottom, it is a right edge.

The following structure is designed to record the information of a rectilinear block:

\section{struct $B$}

\section{\{}

type: HRiB;

$\boldsymbol{B} \boldsymbol{E}^{/ / \mathrm{X}}$ : an ordered set recording the bottom edges from bottom to top;

$\boldsymbol{T E}^{/ / \mathrm{X}}$ : an ordered set recording the top edges from bottom to top;

$\boldsymbol{L} \boldsymbol{E}^{/ / Y}$ : an ordered set recording the left edges from left to right;

$\boldsymbol{R} \boldsymbol{E}^{/ / Y}$ : an ordered set recording the right edges from left to right;

\};

For more clarity, Fig.2 illustrates the four types of edges. As can be seen, $\boldsymbol{a}$ and $\boldsymbol{e}$ are bottom edges; $\boldsymbol{c}, \boldsymbol{i}, \boldsymbol{g}$, and $\boldsymbol{k}$ are top edges; $\boldsymbol{b}, \boldsymbol{f}, \boldsymbol{d}$, and $\boldsymbol{j}$ are left edges; $\boldsymbol{h}$ and $\boldsymbol{l}$ are right edges.

\section{B. Implementation of the Algorithm}

If a block is projected to the $\mathrm{X}$-axis vertically or to the Y-axis horizontally, the blocks in the projection area will affect the bottom-most or the left-most position where this block can move to. In Fig.3, the shadow area is the projection area of $\boldsymbol{A}$. As can be seen, $\boldsymbol{B}, \boldsymbol{C}, \boldsymbol{D}$, and

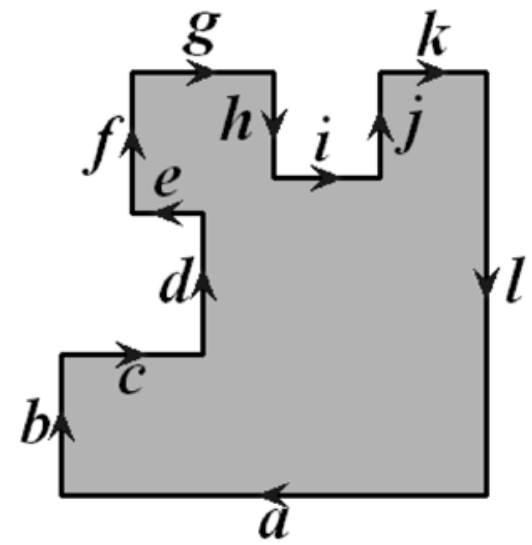

Fig.2. The edges of a rectilinear block 
$\boldsymbol{E}$ are within the projection area, thus, they may affect the moving process of $\boldsymbol{A}$. Because $\boldsymbol{F}$ is outside the projection area, it has no effect on the moving process of $\boldsymbol{A}$. According to this, the following relative positions are defined for two edges.

Definition 5: Let $\boldsymbol{a}^{/ / \mathrm{X}}$ and $\boldsymbol{b}^{/ / \mathrm{X}}$ be two edges parallel to the X-axis. If $\boldsymbol{a}^{/ / \mathrm{X}}$ and $\boldsymbol{b}^{/ / \mathrm{X}}$ satisfy (4), $\boldsymbol{a}^{/ / \mathrm{X}}$ top-overlaps $\boldsymbol{b}^{/ / \mathrm{X}}$; otherwise, $\boldsymbol{a}^{/ / \mathrm{X}}$ un-top-overlaps $\boldsymbol{b}^{/ / \mathrm{X}}$. Let $\boldsymbol{a}^{/ / \mathrm{Y}}$ and $\boldsymbol{b}^{/ / \mathrm{Y}}$ be two edges parallel to the Y-axis. If $\boldsymbol{a}^{/ / Y}$ and $\boldsymbol{b}^{/ / \mathrm{Y}}$ satisfy (5), $\boldsymbol{a}^{/ / Y}$ right-overlaps $\boldsymbol{b}^{/ / Y}$; otherwise, $\boldsymbol{a}^{/ / \mathrm{Y}}$ un-right-overlaps $\boldsymbol{b}^{/ / \mathrm{Y}}$.

$$
\begin{aligned}
& \left(\boldsymbol{a}^{/ / \mathrm{X}}(y) \geq \boldsymbol{b}^{/ / \mathrm{X}}(y)\right) \text { and }\left(\boldsymbol{a}^{/ / \mathrm{X}}\left(x^{R}\right)>\boldsymbol{b}^{/ / \mathrm{X}}\left(x^{L}\right)\right) \text { and }\left(\boldsymbol{a}^{/ / \mathrm{X}}\left(x^{L}\right)<\boldsymbol{b}^{/ / \mathrm{X}}\left(x^{R}\right)\right) \\
& \left(\boldsymbol{a}^{/ / \mathrm{Y}}(x) \geq \boldsymbol{b}^{/ / \mathrm{Y}}(x)\right) \text { and }\left(\boldsymbol{a}^{/ / \mathrm{Y}}\left(y^{T}\right)>\boldsymbol{b}^{/ / \mathrm{Y}}\left(y^{B}\right)\right) \text { and }\left(\boldsymbol{a}^{/ / \mathrm{Y}}\left(y^{B}\right)<\boldsymbol{b}^{/ / \mathrm{Y}}\left(y^{T}\right)\right)
\end{aligned}
$$

Fig.3 shows various top-overlaps and right-overlaps. As can be seen, in Fig.3(a), the bottom edge of $\boldsymbol{A}$ top-overlaps the top edges of $\boldsymbol{B}, \boldsymbol{C}, \boldsymbol{D}$, and $\boldsymbol{E}$; that is, $\boldsymbol{a}$ top-overlaps $\boldsymbol{b}, \boldsymbol{c}, \boldsymbol{d}$, and $\boldsymbol{e}$. Similarly, the right-overlaps are shown in Fig.3(b).

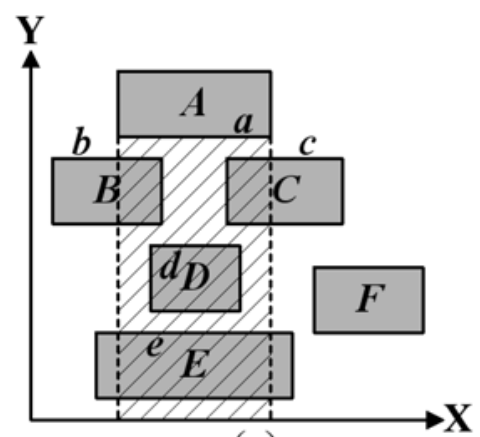

(a)

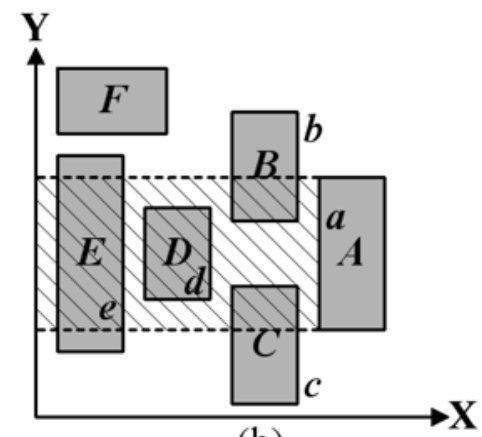

(b)

Fig.3. (a) top-overlaps (b) right-overlaps

With the intrinsic properties of rectilinear blocks in mind, the appropriate position where a rectilinear block can slide to is determined as follows: let $\boldsymbol{B}$ be a rectilinear block. When $\boldsymbol{B}$ slides leftward, if the $i$ th left edge of $\boldsymbol{B}$ right-overlaps some placed edges, then, let CoverRight $X_{i}$ be the $\mathrm{X}$-coordinate of the right-most edge right-overlapped by $\boldsymbol{B}\left(L E_{i}^{/ / \mathrm{Y}}\right)$; otherwise, let CoverRight $X_{i}$ be 0 . Thus, the distance that $\boldsymbol{B}$ can slide leftward is the minimum 
difference between the X-coordinate of $\boldsymbol{B}\left(L E_{i}^{/ / \mathrm{Y}}\right)$ and CoverRight $X_{i}$, where $0 \leq i<\boldsymbol{B}\left(\left|\boldsymbol{L} \boldsymbol{E}^{/ / Y}\right|\right)$. The case when $\boldsymbol{B}$ slides downward similarly uses $\operatorname{CoverTop}_{i}$ instead of CoverRight $X_{i}$. The sets of CoverRight $X_{i}$ and CoverTop $Y_{i}$ are labeled as CoverRightX and CoverTopY, respectively. Algorithm 1 describes the algorithm transforming an MBS to a floorplan.

\section{Algorithm 1: Algorithm transforming an MBS to a floorplan}

Input: $\boldsymbol{M B S}: \mathbf{M B S}=(\pi, I P) \in \mathcal{M B S}$;

Output: $\mathcal{F}(\mathcal{B})$, where $\mathcal{B}=\left\{\boldsymbol{B}_{0}, \boldsymbol{B}_{1}, \ldots, \boldsymbol{B}_{n-1}\right\}$;

$\boldsymbol{B t o T}^{/ / \mathrm{X}}$ and $\mathbf{L t o \boldsymbol { R } ^ { / / Y }}$ denote two ordered sets of edges parallel to the $\mathrm{X}$ - and Y-axis, respectively. They are used to record the top edges from bottom to top and the right edges from left to right of the blocks that have been placed. $\left(x_{\boldsymbol{B}}^{L B}, y_{\boldsymbol{B}}^{L B}\right)$, width $\boldsymbol{B}_{\boldsymbol{B}}$, and height $\boldsymbol{B}_{\boldsymbol{B}}$ are the same as those of Definition 1.

\{

$$
\begin{aligned}
& \left(x_{\pi_{0}}^{L B}, y_{\pi_{0}}^{L B}\right) \leftarrow(0,0) ; \\
& \left(x^{B o x R T}, y^{B o x R T}\right) \leftarrow\left(\text { width }_{\pi_{0}}, \text { height }_{\pi_{0}}\right) ;
\end{aligned}
$$

Add the top edges of $\pi_{0}$ into $\boldsymbol{B t o T}^{/ / \mathrm{X}}$;

Add the right edges of $\pi_{0}$ into $\mathbf{L} \boldsymbol{t o} \boldsymbol{R}^{/ / Y}$;

for $(i=1 ; i<n ; i \leftarrow i+1)$

\{

switch $\left(I P_{i}\right)$

\{

case 0 : 
$\left(x_{\pi_{i}}^{L B}, y_{\pi_{i}}^{L B}\right) \leftarrow\left(0, y^{B o x R T}\right)$

Compute CoverTopY for $\pi_{i}$ from $B$ Bto $\boldsymbol{T}^{/ / \mathrm{X}}$, that is, for each bottom edge of $\pi_{\text {i }}$, find the first edge in $\boldsymbol{B t o} \boldsymbol{T}^{/ / \mathrm{X}}$ from back to front, which is top-overlapped by $\pi_{\mathrm{i}}$, and record its Y-coordinate into CoverTopY;

$y_{\pi_{i}}^{L B} \leftarrow y_{\pi_{i}}^{L B}-\min \left(\left\{\pi_{i}\left(B E_{j}^{/ / \mathrm{X}}(y)\right)-\right.\right.$ CoverTopY $\left.\left._{j} \mid 0 \leq j<\pi_{i}\left(\left|\boldsymbol{B} \boldsymbol{E}^{/ / \mathrm{X}}\right|\right)\right\}\right) ;$

break;

case 1: $\quad$ Similar to case 0 ;

case 2:

$\left(x_{\pi_{i}}^{L B}, y_{\pi_{i}}^{L B}\right) \leftarrow\left(\max \left(0, x^{B o x R T}-w_{i d t h}\right), y^{B o x R T}\right) ;$

CanMove $\leftarrow$ true;

while $($ CanMove $==$ true $)$

\{

Compute CoverTopY for $\pi_{\mathrm{i}}$ from $\boldsymbol{B t o T}^{/ \mathrm{X}}$;

Suppose that the difference between the Y-coordinate of $\pi_{i}\left(B E_{j}^{/ / \mathrm{X}}\right)$ and CoverTop $Y_{j}$ is the smallest, where $0 \leq j<\pi_{i}\left(\left|\boldsymbol{B E} \boldsymbol{E}^{/ / \mathrm{X}}\right|\right)$. If $\pi_{i}\left(B E_{j}^{/ / \mathrm{X}}\right)$ does not top-overlap any edge in $B \boldsymbol{B t o T}^{/ / \mathrm{X}}, k \leftarrow-1$; otherwise, let $B t o T_{k}^{/ / \mathrm{X}}$ be the top-most edge top-overlapped by $\pi_{i}\left(B E_{j}^{/ / \mathrm{X}}\right)$;

$y_{\pi_{i}}^{L B} \leftarrow y_{\pi_{i}}^{L B}-\left(\pi_{i}\left(B E_{j}^{/ / \mathrm{X}}(y)\right)-\operatorname{CoverTop}_{j}\right)$

if $(k \geq 0)$ CoverLeft $X \leftarrow \operatorname{BtoT}_{k}^{/ / X}\left(x^{L}\right)$; else CoverLeft $X \leftarrow 0$;

$k \leftarrow k-1$;

while $\left((k \geq 0)\right.$ and $\left.\left(\operatorname{BtoT}_{k}^{/ / \mathrm{X}}(y)==\pi_{i}\left(B E_{j}^{/ / \mathrm{X}}(y)\right)\right)\right)$

\{ 


$$
\begin{aligned}
& \text { if }\left(\left(\pi_{i}\left(B E_{j}^{/ / \mathrm{X}}(y)\right) \text { top-overlaps } B \text { tot } T_{k}^{/ / \mathrm{X}}\right)\right. \text { and } \\
& \left.\quad\left(B \operatorname{BtoT}_{k}^{/ / \mathrm{X}}\left(x^{L}\right)<\text { CoverLeft } X\right)\right) \text { CoverLeft } X \leftarrow \operatorname{BtoT}_{k}^{/ / \mathrm{X}}\left(x^{L}\right) ; \\
& k \leftarrow k-1 ;
\end{aligned}
$$$$
\text { \} }
$$

Compute CoverRightX for $\pi_{i}$ from $\mathbf{L t o R} \boldsymbol{R}^{/ / Y}$;

Differ $\leftarrow \min \left(\left\{\pi_{i}\left(\operatorname{LE}_{j}^{/ / \mathrm{Y}}(x)\right)-\right.\right.$ CoverRight $\left.\left._{j} \mid 0 \leq j<\pi_{i}\left(\left|\boldsymbol{L} \boldsymbol{E}^{/ / \mathrm{Y}}\right|\right)\right\}\right)$;

if $\left(\pi_{i}\left(B E_{j}^{/ / X}\left(x^{R}\right)\right)\right.$-Differ $>$ CoverLeft X) CanMove $\leftarrow$ false;

else $\left\{\right.$ Differ $\leftarrow \pi_{i}\left(B E_{j}^{/ / X}\left(x^{R}\right)\right)$-CoverLeftX; CanMove $\leftarrow$ true; $\}$

$x_{\pi_{i}}^{L B} \leftarrow x_{\pi_{i}}^{L B}-$ Differ

\}

break;

case 3: Similar to case 2;

\}

Update $\left(x^{B o x R T}, y^{B o x R T}\right)$;

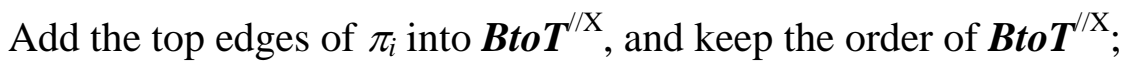

Add the right edges of $\pi_{i}$ into $\mathbf{L} \boldsymbol{t o} \boldsymbol{R}^{/ / Y}$, and keep the order of $\mathbf{L t o R ^ { / / Y }}$;

\}

$\operatorname{Cost}(\mathcal{F}(\mathcal{B})) \leftarrow x^{\text {BoxRT }} \times y^{\text {BoxRT }}$

\}

Algorithm 1 uses two ordered sets of edges, $B \boldsymbol{B t o} \boldsymbol{T}^{/ / \mathrm{X}}$ and $\boldsymbol{L} \boldsymbol{t o} \boldsymbol{R}^{/ / Y}$, to record where the top edges and the right edges have been placed. Thus, it needs only to check $\boldsymbol{B t o T ^ { / / X }}$ and $\boldsymbol{L t o R ^ { / / Y }}$ to find the position where a block can slide to. 
Rectangular blocks are a special case of rectilinear blocks. Thus, Algorithm 1 can handle rectangular blocks directly. However, to improve the performance, Algorithm 1 can be simplified. The structure is simplified to the following form:

\section{struct $B$}

\{

type: $H R a B$ or $S R a B$;

$\left(x^{L B}, y^{L B}\right)$ : the coordinate of the left-bottom corner of $\boldsymbol{B}$;

width, height: the width and height of $\boldsymbol{B}$;

\};

The "switch" part of Algorithm 1 can be easily simplified according to the above structure.

Finally, only the top and right edges are recorded into $\boldsymbol{B t o} \boldsymbol{T}^{/ / \mathrm{X}}$ and $\boldsymbol{L} \boldsymbol{t o} \boldsymbol{R}^{/ / \mathrm{Y}}$, respectively.

Thus, we can see another advantage of $\boldsymbol{B t o T}^{/ / \mathrm{X}}$ and $\mathbf{L} \boldsymbol{t o} \boldsymbol{R}^{/ / \mathrm{Y}}$ : they help the algorithm to be free from the restrictions on the type of blocks that have been placed. Similarly, it is not necessary to know how many blocks have been placed. Thus, rectangular and rectilinear blocks can be handled simultaneously.

Furthermore, we have the following theorem for rectangular blocks:

Theorem 2: The computational complexity of transforming an MBS to a floorplan with rectangular blocks (Algorithm 1) is between linear and quadratic in terms of the number of blocks.

Proof: The main operation of Algorithm 1 is to judge the position of a block in relation to the blocks that have been placed. Because the two ordered sets, $B \boldsymbol{B t o T ^ { / / X }}$ and $\boldsymbol{L} \boldsymbol{t o} \boldsymbol{R}^{/ / Y}$ are used, the operation is changed to that of judging the position of the bottom edge in relation to the 
edges in $\boldsymbol{B t o T ^ { / / X }}$ and the position of the left edge in relation to the edges in $\boldsymbol{L t o R}^{/ / \mathrm{Y}}$.

Because all blocks are rectangular, when placing the ith block, both $\boldsymbol{B t o T ^ { / / X }}$ and $\mathbf{L t o R ^ { / / Y }}$ have (i-1) edges. In the best case, only one judgment needs to be made, thus, the computational complexity of Algorithm 1 is $\mathrm{O}(n)$. In the worst case, $(2 \times[i-1])$ judgments need to be made, and the number of judgments for $n$ blocks is $n(n-1)$. Thus, the computational complexity is $\mathrm{O}\left(n^{2}\right)$.

Theorem 2 shows that, without $\boldsymbol{B t o T}^{/ / \mathrm{X}}$ and $\mathbf{L t o R ^ { / / Y }}$, comparisons with all blocks that have been placed need to be made and the computational complexity is always quadratic. Since most of the preceding edges in $\boldsymbol{B t o T ^ { / / X }}$ and $\boldsymbol{L} \boldsymbol{t o} \boldsymbol{R}^{/ / Y}$ are covered by the following edges and only a small part needs to be compared, the computational complexity is far lower than quadratic.

\section{An Example}

To illustrate the idea of Algorithm 1, the transforming process of an MBS to a floorplan with 10 blocks is shown in Fig.4. $\mathcal{B}=\{\boldsymbol{A}, \boldsymbol{B}, \boldsymbol{C}, \boldsymbol{D}, \boldsymbol{E}, \boldsymbol{F}, \boldsymbol{G}, \boldsymbol{H}, \boldsymbol{I}, \boldsymbol{J}\}$, where $\boldsymbol{I}$ and $\boldsymbol{J}$ are rectilinear blocks. $\boldsymbol{M B S}=(\pi, \boldsymbol{I P})$, where $\pi=(\boldsymbol{E}, \boldsymbol{F}, \boldsymbol{D}, \boldsymbol{J}, \boldsymbol{H}, \boldsymbol{C}, \boldsymbol{A}, \boldsymbol{G}, \boldsymbol{I}, \boldsymbol{B})$ and $\boldsymbol{I P}=(0,1,2,0,3$, $0,2,2,3)$.

$\boldsymbol{E}$ is placed at the left-bottom corner of the first quadrant, as shown in Fig.4(a). In Figs.4(b) and (c), because the initial positions of $\boldsymbol{F}$ and $\boldsymbol{D}$ are 0 and 1, they are placed above and beside $\boldsymbol{E}$, respectively. $\boldsymbol{J}$ is a rectilinear block and its initial position is 2. Because $\boldsymbol{J}$ needs four moves to slide to the final position, each move is shown in Figs.4(d)-(g). In Fig.4(h), because the initial position of $\boldsymbol{H}$ is $0, \boldsymbol{H}$ is placed at 0 and it does not slide. In Fig.4(i), because the initial position of $\boldsymbol{C}$ is $3, \boldsymbol{C}$ is placed at 3 and it slides leftward. $\boldsymbol{A}$ is also placed at the 
initial position and it does not slide, as shown in Fig.4(j). The initial position of both $\boldsymbol{G}$ and $\boldsymbol{I}$ is 2. Thus, they are placed at 2; they first slide downward, and then leftward, as shown in Figs.4(k)-(l). When $\boldsymbol{B}$ is placed at its initial position 3, it slides left, down, and left again, as shown in Figs.4(m)-(o). Finally, an LB-compact floorplan composed of 10 blocks is obtained.

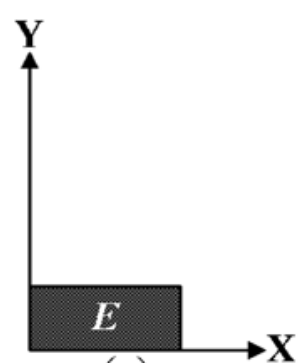

(a)
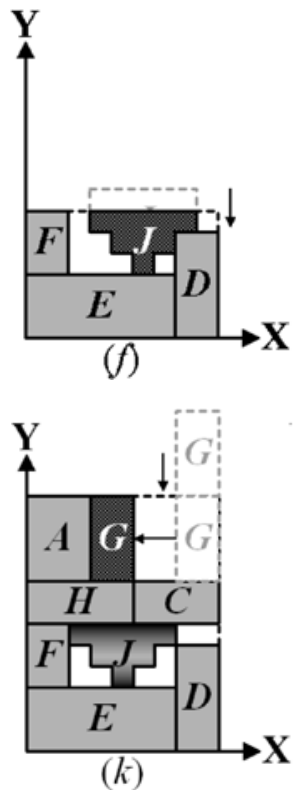
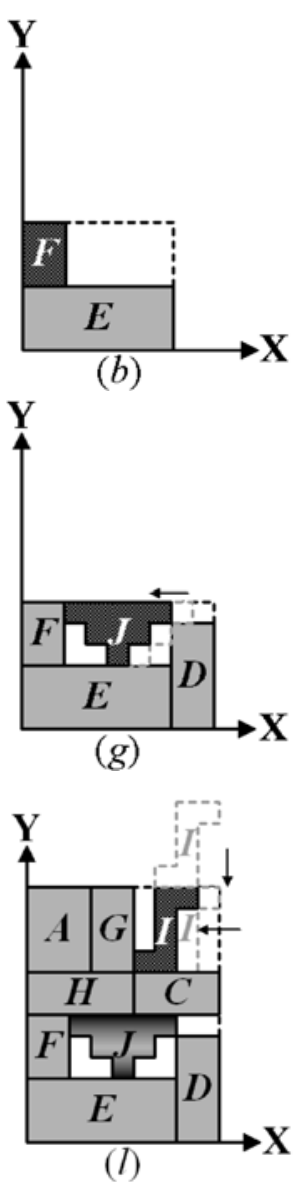

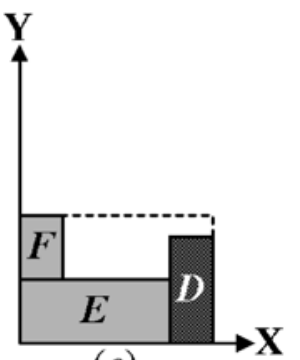

(c)
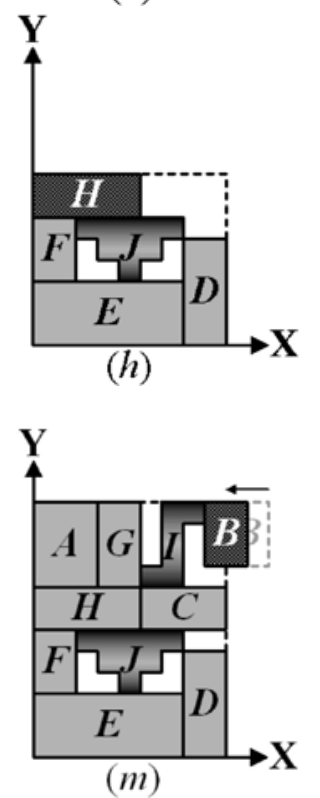
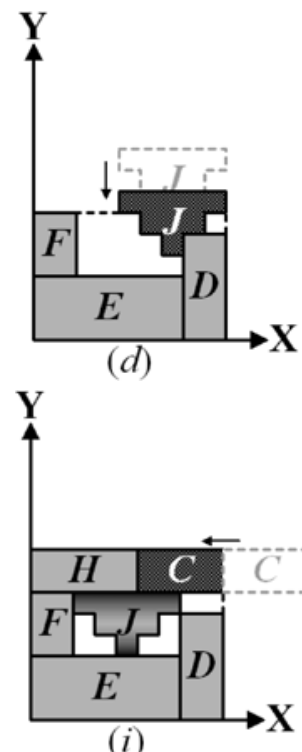

(i)

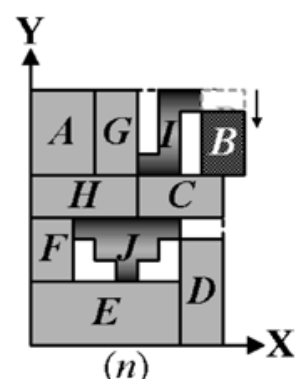

$(n)$
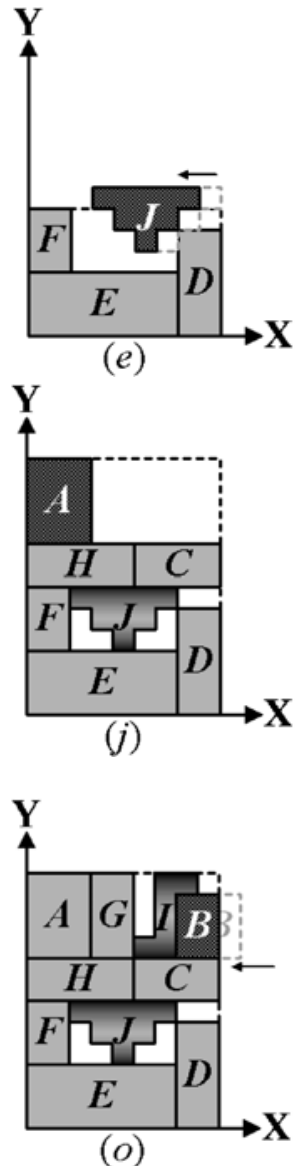

Fig.4. The moving process of each block

\section{Organizational Evolutionary Algorithm based on the Moving Block}

\section{Sequence}

In economics, R. H. Coase explained the sizing and formation of organizations from the framework of transaction costs [43]. This concept was introduced by Wilcox in 1995 to the classifier based on GAs [44]. In a real situation, organizations will compete or cooperate with each other to achieve their purposes. Inspired by this, in our previous works, we proposed the 
framework of the OEA for evolutionary optimization [7]. In the OEA framework, a population is composed of organizations and an organization of members. All evolutionary operators are performed on organizations to simulate the interaction among organizations.

Because the framework of the OEA is general, the problem specific meaning of the organizations and evolutionary operators must be determined before the framework can be used. In the following text, the definition of organizations for floorplanning is first given, and then the evolutionary operators are designed.

\section{A. Definition of Organizations for Floorplanning}

An ordered set $\boldsymbol{S}$ is defined to represent the shape and orientation of each block.

Definition 6: $\boldsymbol{S}$ is an ordered set where the ith element in $\boldsymbol{S}$ corresponds to the $i$ th block. Let $S=\left\{s_{0}, s_{1}, \ldots, s_{n-1}\right\}$, then,

$$
\begin{cases}s_{i} \in\{0,1\} & \text { if } \boldsymbol{B}_{i}(\text { type })=H R a B \\ s_{i} \in\left[\boldsymbol{B}_{i}(\text { minh_w }), \boldsymbol{B}_{i}(\text { maxh_w })\right] & \text { if } \boldsymbol{B}_{i}(\text { type })=S R a B \\ s_{i} \in\{0,1,2,3\} & \text { if } \boldsymbol{B}_{i}(\text { type })=H R i B\end{cases}
$$

where $\boldsymbol{B}_{i}\left(\operatorname{minh} \_w\right)$ and $\boldsymbol{B}_{i}\left(m a x h \_w\right)$ are the minimum and maximum ratios of height to width of $\boldsymbol{B}_{i}$. For an HRaB, $s_{i}$ is equal to 0 or 1 corresponding to rotating the block 0 or 90 degrees clockwise, respectively. For an $\mathrm{SRaB}, s_{i}$ is a real number between minh_w and maxh_w. For an $\mathrm{HRiB}, s_{i}$ is equal to $0,1,2$, or 3 corresponding to rotating the block $0,90,180$, or 270 degrees clockwise, respectively.

Determining the orientations of HRiBs should be based on the previous structure of the rectilinear blocks. When a rectilinear block is rotated, the four types of edges must be recorded again into the structure.

Because the organizations are composed of members, members are defined as follows: 
Definition 7: A Member corresponds to an MBS and is represented as

\section{struct Member}

\{

MBS: the MBS corresponding to this member;

$S$ : the shape and orientation of each block;

$\mathcal{F}(\mathcal{B})$ : the floorplan determined by $\boldsymbol{S}$ and $\boldsymbol{M B S}$;

Cost: $\operatorname{Cost}(\mathcal{F}(\mathcal{B}))$

Trained: a flag used in the evolutionary operators;

\};

Obviously, the smaller the Cost of a member, the better the member is. Thus, an organization is defined as follows:

Definition 8: An organization, $\boldsymbol{O r g}$, is an ordered set, which is composed of more than one member. That is,

$$
\left\{\begin{array}{l}
\text { Org }=\left\{\text { Member }_{0}, \text { Member }_{1}, \ldots, \text { Member }_{m-1}\right\} \quad m \geq 1 \\
\text { Member }_{0}(\text { Cost }) \leq \text { Member }_{1}(\text { Cost }) \leq \ldots \leq \text { Member }_{m-1}(\text { Cost })
\end{array}\right.
$$

\section{B. Evolutionary Operators for Organizations}

On the basis of properties of the MBS, three evolutionary operators are designed for organizations, namely, the splitting operator, the annexing operator, and the training operator.

Splitting operator: This operator is used to prevent the organizations from getting too large. If the size of an organization reaches $\operatorname{Max}_{O S}$, this organization is split with probability 1 where $\operatorname{Max}_{O S}$ is a predefined parameter. Otherwise, this organization is split with a probability proportional to its size. That is, a parent organization will be split into two child organizations, if it satisfies (8), 


$$
\left(|\operatorname{Org}|>\operatorname{Max}_{O S}\right) \text { or }\left\{\left(2 \leq|\operatorname{Org}| \leq \operatorname{Max}_{O S}\right) \text { and }\left(\mathrm{U}(0,1)<|\operatorname{Org}| / N_{\text {Member }}\right)\right\}
$$

where $|\boldsymbol{O r g}|$ is the number of members in $\boldsymbol{O r g}, \mathrm{U}(0,1)$ is a random real number uniformly distributed in 0 to 1 , and $N_{\text {member }}$ is the total number of members of all organizations. Let $\mathbf{O r g}^{P}$ be a parent organization satisfying (8), then, $\boldsymbol{O r g}^{P}$ will be split into two child organizations as follows: $\frac{1}{3}\left|\boldsymbol{O r g}^{P}\right|$ to $\frac{2}{3}\left|\boldsymbol{O r g}^{P}\right|$ members are first randomly selected from $\boldsymbol{O r g}^{P}$ to form one child organization, $\boldsymbol{O r g}^{C_{1}}$, and the remainder forms another child organization, $\boldsymbol{O r g}^{C_{2}}$.

Annexing operator: This operator is used to embody the competition between two organizations. Let two parent organizations be $\operatorname{Org}^{P_{1}}=\left\{\right.$ Member $_{0}^{P_{1}}$, Member $_{1}^{P_{1}}, \ldots$, Member $\left._{m_{1}-1}^{P_{1}}\right\} \quad$ and $\operatorname{Org}^{P_{2}}=\left\{\right.$ Member $_{0}^{P_{2}}$, Member $_{1}^{P_{2}}, \ldots$, Member $\left._{m_{2}-1}^{P_{2}}\right\}$. Because Member $_{0}^{P_{1}}$ and Member $_{0}^{P_{2}}$ are the best ones among $\boldsymbol{O r g}^{P_{1}}$ and $\boldsymbol{O r g}^{P_{2}}$, $\boldsymbol{M e m b e r}_{0}^{P_{1}}($ Cost $)$ and $\boldsymbol{M e m b e r}_{0}^{P_{2}}(\mathrm{Cost})$ are compared. Without loss of the generality, let $\boldsymbol{M e m b e r}_{0}^{P_{1}}($ Cost $)$ be smaller. Then, $\boldsymbol{O r g}^{P_{2}}$ will be annexed by $\boldsymbol{O r g}^{P_{1}}$. That is, $\boldsymbol{O} \boldsymbol{r g}{ }^{P_{1}}$ will increase $m_{2}$ new members, and $\boldsymbol{O} \boldsymbol{r g}{ }^{P_{2}}$ will be removed from the population. The $m_{2}$ new members are generated in two ways: the first uses $\boldsymbol{M e m b e r}_{0}^{P_{1}}$ and those members in the first half of $\boldsymbol{O r g}^{P_{2}}$, labeled as $\operatorname{Generator}_{1}\left(\mathbf{M e m b e r}_{1}, \mathbf{M e m b e r}_{2}, \mathbf{M e m b e r}_{n e w}\right)$, and the second uses the members of $\boldsymbol{O r g}^{P_{1}}$ only, labeled as $\operatorname{Generator}_{2}\left(\right.$ Member, $\left._{\text {Member }}{ }_{n e w}\right)$.

In Generator $_{1}, \quad \operatorname{Member}_{1}(\boldsymbol{M B S})=\left(\pi^{1}, \boldsymbol{I P}^{1}\right), \quad \operatorname{Member}_{1}(\boldsymbol{S})=\left\{s_{0}^{1}, s_{1}^{1}, \ldots, s_{n-1}^{1}\right\}$, $\operatorname{Member}_{2}(\boldsymbol{M B S})=\left(\pi^{2}, \boldsymbol{I P}^{2}\right) \quad, \quad \operatorname{Member}_{2}(\boldsymbol{S})=\left\{s_{0}^{2}, s_{1}^{2}, \ldots, s_{n-1}^{2}\right\} \quad$, $\operatorname{Member}_{\text {new }}(\boldsymbol{M B S})=\left(\pi^{\text {new }}, \boldsymbol{I P}^{\text {new }}\right)$, and $\boldsymbol{M e m b e r}_{\text {new }}(\boldsymbol{S})=\left\{s_{0}^{\text {new }}, s_{1}^{\text {new }}, \ldots, s_{n-1}^{\text {new }}\right\}$. Let $\pi^{\text {new }}$ be equal to $\pi^{1}$, and each element of $\boldsymbol{I P}{ }^{\text {new }}$ to the corresponding one of $\boldsymbol{I P}$ or $\boldsymbol{I P}$ with the same probability. Each element of $\operatorname{Member}_{n e w}(\boldsymbol{S})$ is equal to the corresponding one of $\boldsymbol{M e m b e r}_{1}(\boldsymbol{S})$ 
or $\operatorname{Member}_{2}(\boldsymbol{S})$ with the same probability for HRaBs and HRiBs, and to the results of the real number crossover with $\operatorname{Member}_{1}(\boldsymbol{S})$ and $\boldsymbol{M e m b e r}_{2}(\boldsymbol{S})$ for SRaBs. That is,

$$
\begin{aligned}
& \pi^{\text {new }}=\pi^{1} \text { and } I P_{i}^{\text {new }}= \begin{cases}I P_{m}^{1} & \text { if }\left(\pi_{m}^{1}=\pi_{l}^{2}\right) \text { and }(\mathrm{U}(2)=0) \\
I P_{l}^{2} & \text { if }\left(\pi_{m}^{1}=\pi_{l}^{2}\right) \text { and }(\mathrm{U}(2)=1)\end{cases}
\end{aligned}
$$

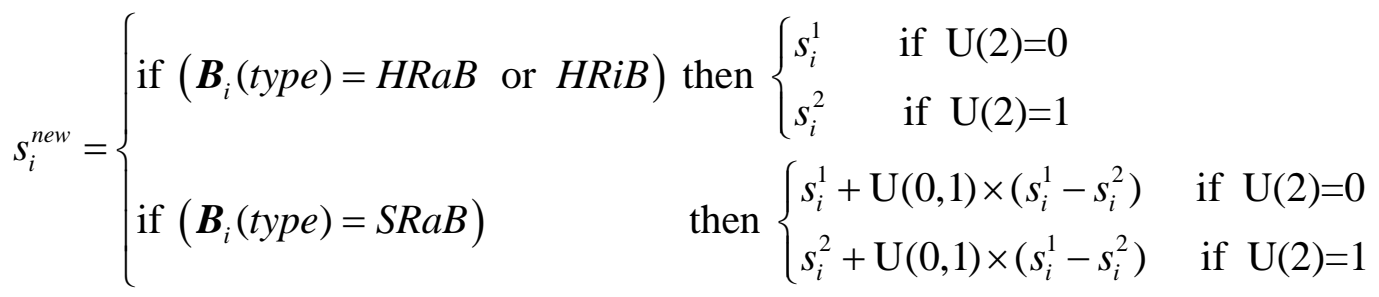

where $0 \leq i, m, l<n$, and $\mathrm{U}(x)$ is a random integer uniformly distributed in 0 to $x-1$. What should be noted is that $\pi_{i}^{1}$ and $\pi_{i}^{2}$ may denote different blocks, thus, the same blocks in the two permutations must be identified first, that is, $\pi_{m}^{1}=\pi_{l}^{2}$ in (9). Finally, if $\boldsymbol{M e m b e r}_{2}(\operatorname{Cost})$ is smaller than $\mathbf{M e m b e r}_{n e w}($ Cost $)$, Member $_{\text {new }}$ is replaced by $\mathbf{M e m b e r}_{2}$.

In Generator $_{2}, \quad \operatorname{Member}(\operatorname{MBS})=(\pi, \boldsymbol{I P}), \quad \operatorname{Member}(\boldsymbol{S})=\left\{s_{0}, s_{1}, \ldots, s_{n-1}\right\} \quad$, $\operatorname{Member}_{\text {new }}(\boldsymbol{M B S})=\left(\boldsymbol{\pi}^{\text {new }}, \boldsymbol{I P}{ }^{\text {new }}\right)$, and $\boldsymbol{M e m b e r}_{\text {new }}(\boldsymbol{S})=\left\{s_{0}^{\text {new }}, s_{1}^{\text {new }}, \ldots, s_{n-1}^{\text {new }}\right\}$. First, assign Member to $\mathbf{M e m b e r}_{\text {new }}$, and then for each element of $\pi^{\text {new }}, \boldsymbol{I P}^{\text {new }}$, and $\operatorname{Member}_{\text {new }}(\boldsymbol{S})$, perform (11) if $\mathrm{U}(0,1)<1 / n$.

$$
\left\{\begin{array}{l}
\pi_{i}^{\text {new }} \leftrightarrow \pi_{j}^{\text {new }} \quad \text { where } j=\mathrm{U}(n) \text { and }(i \neq j) \\
I P_{i}^{\text {new }}=\mathrm{U}(4) \quad \text { where } \mathrm{U}(4) \neq I P_{i}^{\text {new }}
\end{array}\right.
$$

where $0 \leq i<n, \mathrm{G}(0, \sigma)$ is a Gauss random real number distributed in 0 to $\sigma$, and $\sigma=\left[\boldsymbol{B}_{i}(\right.$ maxh_w $)-\boldsymbol{B}_{i}($ minh_w) $\left.)\right] / 100$.

Let the new $m_{2}$ members of $\boldsymbol{O r g}^{P_{1}}$ be $\left\{\right.$ Member $_{0}^{C}$, Member $\left._{1}^{C}, \ldots, \boldsymbol{M e m b e r}_{m_{2}-1}^{C}\right\}$. Then, 


$$
\left\{\begin{array}{lc}
\text { Generator }_{1}\left(\text { Member }_{0}^{P_{1}}, \text { Member }_{i}^{P_{2}}, \text { Member }_{i}^{C}\right) & 0 \leq i \leq\left\lfloor m_{2} / 2\right\rfloor \\
\text { Generator }_{2}\left(\text { Member }_{i-\left\lfloor m_{2} / 2\right\rfloor \bmod m_{1}}^{P_{1}}, \text { Member }_{i}^{C}\right) & \left\lfloor m_{2} / 2\right\rfloor<i<m_{2}
\end{array}\right.
$$

where $\left\lfloor m_{2} / 2\right\rfloor$ is the maximum integer smaller than $m_{2} / 2$, and "mod" denotes getting the

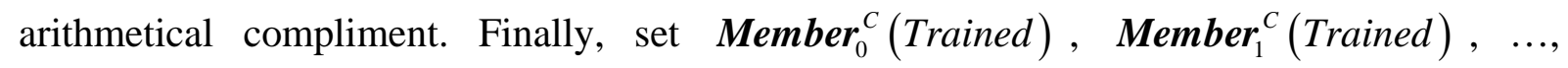
Member $_{m_{2}-1}^{C}($ Trained $)$ to false.

Training operator: This operator is used to improve qualities of members. Let Org be the parent organization. Then, select the first member in Org whose Trained is false or the first member in $\mathbf{O r g}$ when Trained of all members is true. Let the information of the selected member be $\operatorname{Member}(\boldsymbol{M B S})=(\pi, \boldsymbol{I P})$ and $\operatorname{Member}(\boldsymbol{S})=\left\{s_{0}, s_{1}, \ldots, s_{n-1}\right\}$. The training process consists of three parts: $\boldsymbol{\pi}, \boldsymbol{I P}$, and $\boldsymbol{S}$. These three parts are independent, thus, the order to perform them is also random.

Training $\pi$ : For $\pi_{i}(0 \leq i<n)$, select another element in $\pi$ to exchange with it. If Member(Cost) is smaller than before, the exchange is accepted; otherwise, the exchange is given up. This process is repeated 5 times.

Training IP: Assign the three other initial positions to each $I P_{i}(1 \leq i<n)$, one by one. If Member(Cost) is improved, then, the corresponding initial position is accepted.

Training $\boldsymbol{S}$ : For hard blocks, assign other values to $s_{i}$, one by one; for soft blocks, assign values to $s_{i}$ as follows:

$$
s_{i}= \begin{cases}\boldsymbol{B}^{i}\left(\text { minh } \_w\right)+\text { Range } \times j+\mathrm{U}(0,1) \times \text { Range } & \text { if } \mathrm{U}(2)=0 \\ s_{i}+\mathrm{G}_{j}(0, \sigma) & \text { if } \mathrm{U}(2)=1\end{cases}
$$

where Range $=\left[\boldsymbol{B}_{i}\left(\operatorname{maxh} \_w\right)-\boldsymbol{B}_{i}\left(\operatorname{minh} \_w\right)\right] / 5 \quad(0 \leq j \leq 4)$, and $\mathrm{G}_{j}$ denotes the Gauss random number which changes depending on $j$. If the new Member(Cost) is smaller than before, it is accepted; otherwise, it is given up. 
After performing all the three parts, set Member(Trained) to true. Finally, adjust the position of Member in $\mathbf{O r g}$ according to Member(Cost).

The three operators have different functions. The annexing operator embodies the competition between organizations. The organizations with low quality are annexed by the organizations with high quality so that there is more space for the organizations with high potential to develop. The splitting operator controls the size of each organization so that the computational cost is distributed more reasonably. The training operator is the major way that can improve the quality of an organization.

\section{Implementation of the Algorithm}

According to the framework of the OEA, in each generation, the size of each organization in the population is first checked. If the size of an organization satisfies (8), then, the splitting operator is performed on this organization. After that, two organizations are randomly selected from the population to perform the annexing operator until the number of organizations in the population is less than 2. Finally, the training operator is performed on each organization of the next generation. Thus, the population evolves generation by generation, and the solution is the floorplan of the best member among all organizations at the end of the evolutionary process. The details are shown in Algorithm 2.

\section{Algorithm 2: Organizational evolutionary algorithm based on moving block sequence}

Input: $\operatorname{Max}_{O S}, N_{\text {Member }}$ : Predefined parameters;

Output: $\mathcal{F}(\mathcal{B})$, where $\mathcal{B}=\left\{\boldsymbol{B}_{0}, \boldsymbol{B}_{1}, \ldots, \boldsymbol{B}_{n-1}\right\}$;

$\boldsymbol{O r g}_{i}^{t}$ is the ith organization in the population of the $t$ th generation, $\boldsymbol{P}^{t}$. 
Generate $N_{\text {Member }}$ members randomly;

Compute the cost of each member, and set Trained of each member to false;

Use each member to form an organization; //As a result, the initial population, $\boldsymbol{P}^{0}$,

$$
\text { consists //of } N_{\text {Member }} \text { organizations. }
$$

$t \leftarrow 0 ; \quad$ CanTrain $\leftarrow$ true;

while (Stop conditions are not satisfied)

\{

for $\left(i=0 ; i<\left|\boldsymbol{P}^{t}\right| ; i \leftarrow i+1\right)$

\section{\{}

if $\left(\boldsymbol{O r g}_{i}^{t}\right.$ satisfies (8))

\{

Split $\boldsymbol{O r g}_{i}^{t}$ according to the splitting operator;

Add $\boldsymbol{O r g}^{C_{1}}$ and $\boldsymbol{O r g}^{C_{2}}$ into $\boldsymbol{P}^{t+1}$;

Delete $\boldsymbol{O r g}_{i}^{t}$ from $\boldsymbol{P}^{t}$;

\}

\}

while $\left(\left|\boldsymbol{P}^{t}\right|>1\right)$

\{

Select two organizations, $\boldsymbol{O r g}_{1}$ and $\boldsymbol{O} \boldsymbol{r g}$, from $\boldsymbol{P}^{t}$ randomly;

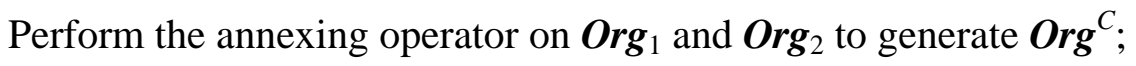

Add $\boldsymbol{O} \boldsymbol{r g}{ }^{C}$ into $\boldsymbol{P}^{t+1}$, and delete $\boldsymbol{O} \boldsymbol{r g} \boldsymbol{g}_{1}$ and $\boldsymbol{O} \boldsymbol{r g} \boldsymbol{r}_{2}$ from $\boldsymbol{P}^{t}$; 


\section{\}}

if $\left(\left|\boldsymbol{P}^{t}\right|==1\right)$ Move $\operatorname{Org}_{0}^{t}$ into $\boldsymbol{P}^{t+1}$;

if $($ CanTrain $==$ true $)$

\{

CanTrain $\leftarrow$ false;

for $\left(i=0 ; i<\left|\boldsymbol{P}^{t+1}\right| ; i \leftarrow i+1\right)$

\{

Perform the training operator on $\mathbf{O r g}_{i}^{t+1}$;

if (the cost of the select member from $\mathbf{O r g}_{i}^{t+1}$ decreases) CanTrain $\leftarrow$ true;

\}

\}

$t \leftarrow t+1$

\}

Set $\mathcal{F}(\mathcal{B})$ of the best member among all organizations in $P^{t}$ to $\mathcal{F}(\mathcal{B})$;

\section{\}}

In the early stage of the evolutionary process, qualities of all members are low, thus, the training operator can dramatically improve the quality of the whole population. But after a period of evolution, qualities of most members are above a particular level and the training operator becomes less effective. To reduce the computational cost, when all members selected from organizations cannot be improved by the training operator in some generation, then, this operator is no longer used in the remaining evolutionary process. 
Because organizations in the OEA consist of members that are similar to individuals used in traditional EAs, one might argue that organizations are similar to subpopulations in coarse-grained parallel EAs (CGP-EAs) [45]-[47]. There are several differences between the OEA and CGP-EAs. First, the number of subpopulations and the size of each subpopulation of CGP-EAs are fixed during the evolutionary process, whereas the number of organizations and the size of each organization of the OEA change from generation to generation. Second, the major interaction among subpopulations in CGP-EAs is to copy better-performing or random individuals to neighboring subpopulations at regular intervals, whereas the interaction among organizations is especially designed and embodied through the evolutionary operators. That is, the OEA can be viewed as using additional operators in the migration process.

However, from another viewpoint, the OEA can be considered a kind of CGP-EAs. Because CGP-EAs are parallel techniques, they often present the same problem as the traditional EAs do. But since the OEA takes inspiration from simulating the interaction among organizations, the following experimental results show that the OEA obtains good performances for various floorplanning problems with a wide range of size. Thus, the OEA can be viewed as a successful development of CGP-EAs.

\section{Experimental Studies}

The experiments consist of three parts: floorplanning problems (i) with hard rectangular blocks; (ii) with soft rectangular blocks; and (iii) with hybrid blocks including both soft rectangular and hard rectilinear blocks. All problems come from MCNC and GSRC benchmarks [48]. The first part uses 21 problems, which are shown in Table I, and the number of blocks increases from 9 to 300. The second part uses 17 problems, which are also 
shown in Table I because they are transformed from the problems used in the first part, and the number of blocks increases from 9 to 100. The third part uses 6 problems, which are shown in Table II, and the number of blocks increases from 30 to 100.

Table I Problems used in the first and second parts of experiments

\begin{tabular}{|c|c|c|c|c|c|}
\hline Problem name & Dataset & \#Blocks & minh_w & maxh_w & Area of all blocks \\
\hline apte & MCNC & 9 & 0.5 & 2.0 & 46.561628 \\
\hline xerox & MCNC & 10 & 0.5 & 2.0 & 19.350296 \\
\hline hp & MCNC & 11 & 0.5 & 2.0 & 8.830584 \\
\hline ami33 & MCNC & 33 & 0.5 & 2.0 & 1.156449 \\
\hline ami49 & MCNC & 49 & 0.5 & 2.0 & 35.445424 \\
\hline $\mathrm{n} 10 \mathrm{a}$ & GSRC & 10 & 0.3 & 3.0 & 22.1679 \\
\hline n10b & GSRC & 10 & 0.3 & 3.0 & 22.1177 \\
\hline $\mathrm{n} 10 \mathrm{c}$ & GSRC & 10 & 0.3 & 3.0 & 22.8770 \\
\hline n30a & GSRC & 30 & 0.3 & 3.0 & 20.8591 \\
\hline n30b & GSRC & 30 & 0.3 & 3.0 & 19.7781 \\
\hline n30c & GSRC & 30 & 0.3 & 3.0 & 22.2522 \\
\hline n50a & GSRC & 50 & 0.3 & 3.0 & 19.8579 \\
\hline n50b & GSRC & 50 & 0.3 & 3.0 & 20.3053 \\
\hline $\mathrm{n} 50 \mathrm{c}$ & GSRC & 50 & 0.3 & 3.0 & 20.1512 \\
\hline $\mathrm{n} 100 \mathrm{a}$ & GSRC & 100 & 0.3 & 3.0 & 17.9501 \\
\hline $\mathrm{n} 100 \mathrm{~b}$ & GSRC & 100 & 0.3 & 3.0 & 16.0126 \\
\hline $\mathrm{n} 100 \mathrm{c}$ & GSRC & 100 & 0.3 & 3.0 & 17.1966 \\
\hline n200a & GSRC & 200 & - & - & 17.5696 \\
\hline n200b & GSRC & 200 & - & - & 17.4593 \\
\hline n200c & GSRC & 200 & - & - & 17.0129 \\
\hline n300a & GSRC & 300 & - & - & 27.3170 \\
\hline
\end{tabular}

The parameters of the MBS-OEA are set as follows: $M a x_{O S}=20$ and $N_{\text {Member }}=1000$. The number of evolutionary generations is set to 1000 when $n \leq 100,2000$ when $n=200$, and 3000 when $n=300$. The measures to evaluate qualities of solutions are the area of minimum bounding rectangle (Area) and Area Ratio,

$$
\text { Area Ratio }=\frac{\text { Area of all blocks }}{\text { Area of minimum bounding rectangle }} \times 100 \%
$$

The MBS-OEA runs 10 times independently on each problem and all experiments are carried out on an IBM IntelliStation Z Pro Type 6221 with 1GB memory. 
Table II Problems used in the third part of experiments

\begin{tabular}{c|c|c|c|c|c|c}
\hline \multirow{2}{*}{ Problem name } & \multirow{2}{*}{ Dataset } & \multicolumn{3}{|c|}{ Soft rectangular blocks } & \#Hard rectilinear & Area of all \\
\cline { 3 - 5 } & & \#Blocks & minh_w & maxh_w & 3 & blocks \\
\hline \hline n030_10 & GSRC & 27 & 0.3 & 3.0 & 3 & 23.6589 \\
\hline n030_20 & GSRC & 24 & 0.3 & 3.0 & 6 & 25.7019 \\
\hline n050_10 & GSRC & 45 & 0.3 & 3.0 & 5 & 23.9720 \\
\hline n050_20 & GSRC & 40 & 0.3 & 3.0 & 10 & 25.9063 \\
\hline n100_10 & GSRC & 90 & 0.3 & 3.0 & 10 & 18.3759 \\
\hline n100_20 & GSRC & 80 & 0.3 & 3.0 & 20 & 19.9165 \\
\hline
\end{tabular}

\section{A. Floorplanning Problems with Hard Rectangular Blocks}

The results of the MBS-OEA are shown in Table III. To illustrate the solutions, the best floorplan found for n300a is shown in Fig.5(a). As can be seen, the best value of the Area Ratios is larger than $90 \%$ for all the 21 problems and larger than $97 \%$ for the problems with no more than 50 blocks. For the problems with no more than 100 blocks, the average value of the Area Ratios is larger than 92\%. Although the average value of the Area Ratios is a bit small for the problems with 200 or 300 blocks, it is still in the range of $88-91 \%$. Thus, the MBS-OEA obtains a good performance in both the best and the average values of the Area Ratios. The results show that the MBS-OEA not only has a strong ability in searching the optima, but also has a stable performance. Moreover, the MBS-OEA finds high quality floorplans for the problems with 200 or 300 blocks, which illustrates that the MBS-OEA is competent for solving large-scale problems.

Table III Results of the MBS-OEA on floorplanning problems with hard rectangular blocks

\begin{tabular}{|c|c|c|c|c|c|}
\hline \multirow{2}{*}{ Problem name } & \multicolumn{2}{|c|}{ Area } & \multicolumn{2}{|c|}{ Area Ratio (\%) } & \multirow{2}{*}{$\begin{array}{c}\text { Average times } \\
\text { (s) }\end{array}$} \\
\hline & Best & Average & Best & Average & \\
\hline apte & 46.924848 & 46.924848 & 99.23 & 99.23 & 0.5 \\
\hline xerox & 19.795608 & 19.879554 & 97.75 & 97.34 & 1.1 \\
\hline hp & 8.947008 & 8.947008 & 98.70 & 98.70 & 1.7 \\
\hline ami33 & 1.175363 & 1.206013 & 98.39 & 95.91 & 45.0 \\
\hline ami49 & 36.350160 & 37.040805 & 97.51 & 95.70 & 173.2 \\
\hline $\mathrm{n} 10 \mathrm{a}$ & 22.6092 & 22.8031 & 98.05 & 97.22 & 0.9 \\
\hline n10b & 22.5585 & 22.7450 & 98.05 & 97.24 & 1.2 \\
\hline
\end{tabular}




\begin{tabular}{c|c|c|c|c|c}
\hline n10c & 23.1804 & 23.3698 & 98.69 & 97.89 & 1.1 \\
\hline n30a & 21.4600 & 21.7734 & 97.20 & 95.81 & 25.6 \\
\hline n30b & 20.1150 & 20.4226 & 98.33 & 96.85 & 29.4 \\
\hline n30c & 22.8242 & 23.2908 & 97.49 & 95.55 & 25.5 \\
\hline n50a & 20.4140 & 20.8561 & 97.28 & 95.22 & 123.2 \\
\hline n50b & 20.9271 & 21.2932 & 97.03 & 95.37 & 121.0 \\
\hline n50c & 20.7564 & 21.2032 & 97.08 & 95.05 & 137.0 \\
\hline n100a & 18.9832 & 19.3208 & 94.56 & 92.91 & 1706.1 \\
\hline n100b & 16.9158 & 17.3306 & 94.66 & 92.40 & 1065.4 \\
\hline n100c & 18.2648 & 18.5742 & 94.15 & 92.59 & 1319.0 \\
\hline n200a & 19.2384 & 19.5258 & 91.33 & 89.99 & 12114.1 \\
\hline n200b & 19.0554 & 19.3817 & 91.62 & 90.09 & 14217.2 \\
\hline n200c & 18.7600 & 18.9976 & 90.69 & 89.56 & 11210.7 \\
\hline n300a & 30.2412 & 30.7492 & 90.33 & 88.84 & 80984.1 \\
\hline
\end{tabular}

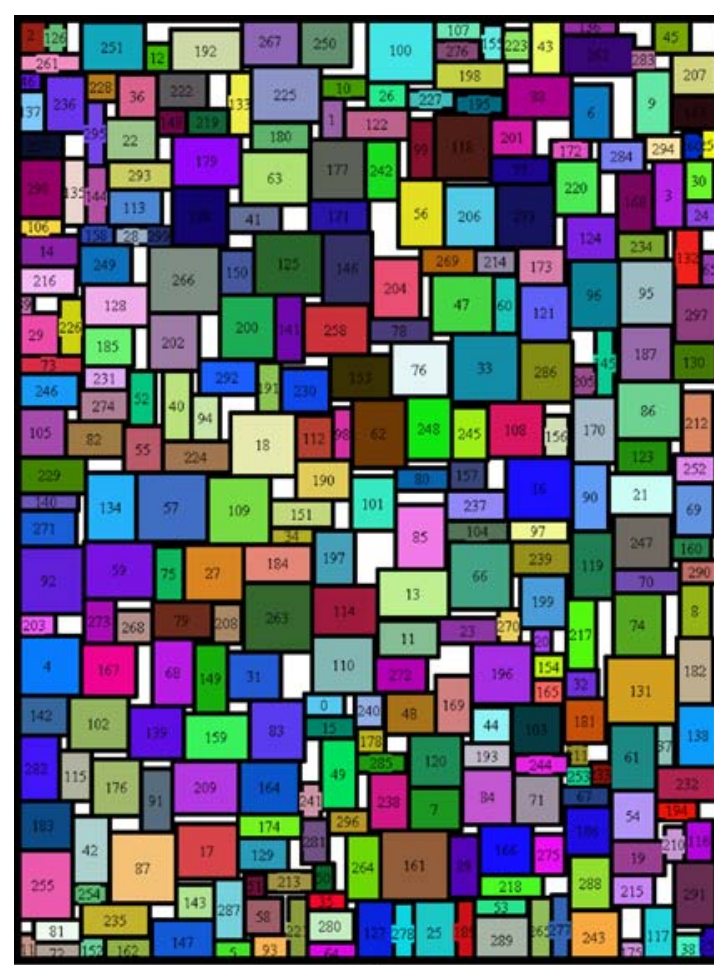

(a)

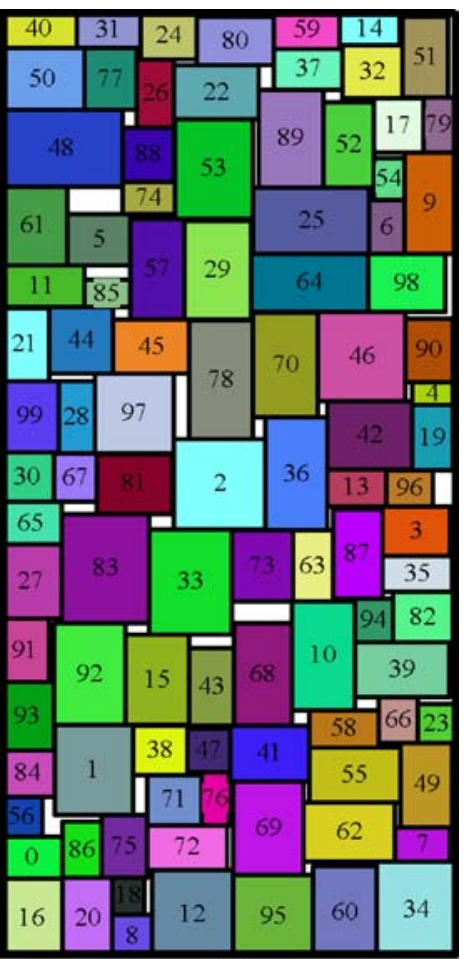

(b) 


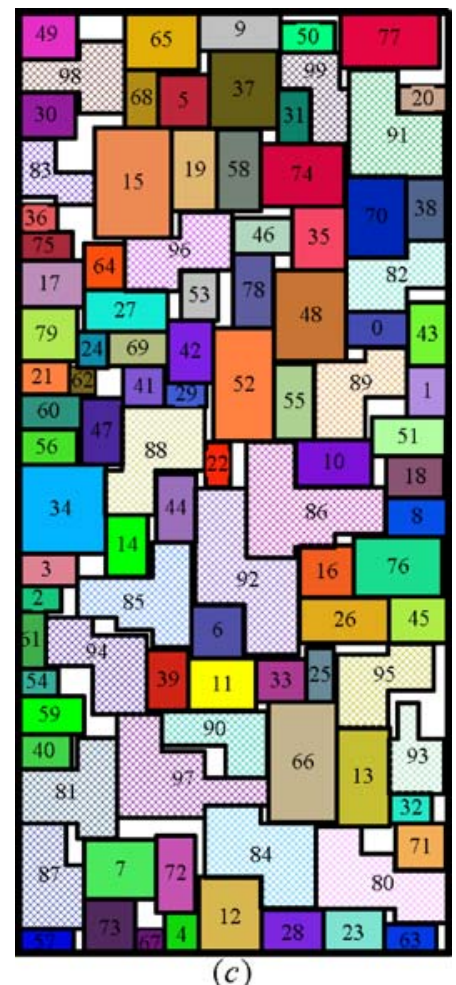

Fig.5. The best floorplans found by the MBS-OEA: (a) n300a (b) n100c (c) n100_20

Table IV compares the results of the MBS-OEA with those of 9 existing well-designed algorithms on the problems from MCNC. The 9 algorithms are the $O$-tree [9], the enhanced $O$-tree [49], the $B^{*}$-tree [10], the TCG [3], the TCG-S [4], the Fast-SP [50], the Enhanced Q-seq [51], the TBS [12], and the CS [5]. The running environments of these algorithms are different and the Times given in Table IV are for references only. The running environment of the $O$-tree, the Enhanced $O$-tree, the TCG, the TCG-S, the Enhanced Q-seq, and the CS is Sun Sparc Ultra60; that of the $B^{*}$-tree and the Fast-SP is Sun Sparc Ultra-I; and that of the TBS is $1400 \mathrm{MHz}$ Intel Xeon PC. In general, the computational cost of the MBS-OEA is in the average level among the 10 algorithms.

Table IV Comparison between the MBS-OEA and 9 existing algorithms on MCNC benchmarks with hard rectangular blocks

\begin{tabular}{|c|c|c|c|c|c|c|c|c|c|c|}
\hline & \multicolumn{2}{|c|}{ apte } & \multicolumn{2}{|c|}{ xerox } & \multicolumn{2}{|c|}{ hp } & \multicolumn{2}{|c|}{ ami33 } & \multicolumn{2}{|c|}{ ami49 } \\
\hline & Area & $\begin{array}{l}\text { Time } \\
\text { (s) }\end{array}$ & Area & $\begin{array}{l}\text { Time } \\
\text { (s) }\end{array}$ & Area & $\begin{array}{c}\text { Time } \\
\text { (s) }\end{array}$ & Area & $\begin{array}{l}\text { Time } \\
\text { (s) }\end{array}$ & Area & $\begin{array}{c}\text { Time } \\
\text { (s) }\end{array}$ \\
\hline O-Tree & 47.1 & 38 & 20.1 & 118 & 9.21 & 57 & 1.25 & 1430 & 37.6 & 7428 \\
\hline
\end{tabular}




\begin{tabular}{l|l|r||r|r||l|r||l|r||r|r}
\hline Enhanced O-Tree & 46.92 & 11 & 20.21 & 38 & 9.16 & 19 & 1.24 & 118 & 37.73 & 406 \\
\hline B $^{*}$-Tree & 46.92 & 7 & 19.83 & 25 & 8.947 & 55 & 1.27 & 3417 & 36.80 & 4752 \\
\hline TCG & 46.92 & 1 & 19.83 & 18 & 8.947 & 20 & 1.20 & 306 & 36.77 & 434 \\
\hline TCG-S & 46.92 & 1 & 19.80 & 5 & 8.947 & 7 & 1.185 & 84 & 36.40 & 369 \\
\hline Fast-SP & 46.92 & 1 & 19.80 & 14 & 8.947 & 6 & 1.205 & 20 & 36.5 & 31 \\
\hline Enhanced Q-seq & 46.92 & 0.35 & 19.93 & 3.6 & 9.03 & 3.5 & 1.194 & 40 & 36.75 & 57 \\
\hline TBS & 47.44 & 0.86 & 19.78 & 1.3 & $8.48^{\#}$ & 0.76 & 1.196 & 1.26 & 36.89 & 2.55 \\
\hline CS & 46.92 & 1 & 19.83 & 54 & 8.947 & 6 & 1.18 & 530 & 36.28 & 851 \\
\hline MBS-OEA & 46.92 & 0.5 & 19.80 & 1.1 & 8.947 & 1.7 & 1.175 & 45 & 36.35 & 173.2 \\
\hline
\end{tabular}

" Smaller than the total area of all blocks, a wrong solution

As can be seen, for apte, the Area of the MBS-OEA is as small as those of 7 other algorithms. For xerox, the Areas of the MBS-OEA, the TCG-S, and the Fast-SP are the same, and are slightly larger than that of the TBS. For hp, although the Area of the TBS is the smallest (8.48), it is a wrong solution since it is even smaller than the total area of all blocks (8.83). For ami33, the Area of the MBS-OEA is the smallest among the 10 algorithms. For ami49, the Area of the MBS-OEA is slightly larger than that of the CS, but smaller than those of 8 other algorithms. To summarize, the MBS-OEA obtains a better performance, especially for the two larger problems, ami33 and ami49.

Table $\mathrm{V}$ compares the results of the MBS-OEA with those of website [48] on the problems from GSRC. As can be seen, the Areas of the MBS-OEA on 15 of the 16 problems are smaller than those of [48]. Only for n200c, the Area of the MBS-OEA is slightly larger.

Table V Comparison between the MBS-OEA and [48] on GSRC benchmarks with hard rectangular blocks (Area)

\begin{tabular}{c|c|c|c|c|c|c|c|c}
\hline & $\mathrm{n} 10 \mathrm{a}$ & $\mathrm{n} 10 \mathrm{~b}$ & $\mathrm{n} 10 \mathrm{c}$ & $\mathrm{n} 30 \mathrm{a}$ & $\mathrm{n} 30 \mathrm{~b}$ & $\mathrm{n} 30 \mathrm{c}$ & $\mathrm{n} 50 \mathrm{a}$ & $\mathrm{n} 50 \mathrm{~b}$ \\
\hline [48] & 23.5578 & 23.6720 & 24.3600 & 22.6350 & 21.1692 & 24.2942 & 21.6524 & 22.1112 \\
\hline MBS-OEA & 22.6092 & 22.5585 & 23.1804 & 21.4600 & 20.1150 & 22.8242 & 20.4140 & 20.9271 \\
\hline \hline & $\mathrm{n} 50 \mathrm{c}$ & $\mathrm{n} 100 \mathrm{a}$ & $\mathrm{n} 100 \mathrm{~b}$ & $\mathrm{n} 100 \mathrm{c}$ & $\mathrm{n} 200 \mathrm{a}$ & $\mathrm{n} 200 \mathrm{~b}$ & $\mathrm{n} 200 \mathrm{c}$ & $\mathrm{n} 300 \mathrm{a}$ \\
\hline [48] & 21.8040 & 19.8492 & 17.7514 & 18.7726 & 19.4340 & 19.2888 & 18.6966 & 30.4703 \\
\hline MBS-OEA & 20.7564 & 18.9832 & 16.9158 & 18.2648 & 19.2384 & 19.0554 & 18.7600 & 30.2412 \\
\hline
\end{tabular}

\section{B. Floorplanning Problems with Soft Rectangular Blocks}

The results of the MBS-OEA are shown in Table VI. The best floorplan found for n100c 
is shown in Fig.5(b). As can be seen, the best value of the Area Ratios for the problems with about 10 blocks achieves up to $100 \%$. Both the best and the average values of the Area Ratios for the problems with no more than 50 blocks are larger than 98\%. Even for the problems with 100 blocks, the average value of the Area Ratios is also larger than 94\%. Thus, the MBS-OEA also shows a stable good performance for floorplanning problems with soft rectangular blocks.

Table VII compares the results of the MBS-OEA with those of two existing algorithms, [52] and the CBL [11]. The running environments of the algorithm in [52] and the CBL are 600-MHz Pentium III and Sun Sparc Ultra60, respectively. As can be seen, for all the 5 problems, the Areas of the MBS-OEA are smaller than those of [52] and the CBL.

\section{Floorplanning Problems with Hybrid Blocks}

The results of the MBS-OEA are shown in Table VIII. The best floorplan found for n100_20 is shown in Fig.5(c). As can be seen, for all the 6 problems, both the best and the average values of the Area Ratios are larger than $90 \%$.

Table VI Results of the MBS-OEA on floorplanning problems with soft rectangular blocks

\begin{tabular}{c|c|c|c|c|c}
\hline \multirow{2}{*}{ Problem name } & \multicolumn{2}{|c|}{ Area } & \multicolumn{2}{c|}{ Area Ratio (\%) } & Average times \\
& Best & Average & Best & Average & 18.5 \\
\hline \hline apte & 46.561634 & 46.569551 & 100.00 & 99.98 & 21.2 \\
\hline xerox & 19.350296 & 19.368267 & 100.00 & 99.91 & 26.1 \\
\hline hp & 8.830584 & 8.834488 & 100.00 & 99.96 & 361.4 \\
\hline ami33 & 1.160467 & 1.165469 & 99.65 & 99.23 & 890.3 \\
\hline ami49 & 35.774650 & 35.918632 & 99.08 & 98.68 & 23.1 \\
\hline n10a & 22.1686 & 22.1780 & 100.00 & 99.95 & 17.1 \\
\hline n10b & 22.1177 & 22.1186 & 100.00 & 100.00 & 22.0 \\
\hline n10c & 22.8770 & 22.8911 & 100.00 & 99.94 & 300.7 \\
\hline n30a & 20.9244 & 21.0313 & 99.69 & 99.18 & 296.7 \\
\hline n30b & 19.8682 & 19.9347 & 99.55 & 99.22 & 284.7 \\
\hline n30c & 22.2838 & 22.4308 & 99.86 & 99.21 & 949.7 \\
\hline n50b & 20.0882 & 20.2164 & 98.85 & 98.23 & 1077.3 \\
\hline
\end{tabular}




\begin{tabular}{l|l|l|l|l|l}
\hline n50c & 20.3789 & 20.5352 & 98.88 & 98.13 & 1022.3 \\
\hline n100a & 18.8893 & 19.0752 & 95.03 & 94.11 & 6440.4 \\
\hline n100b & 16.8116 & 16.9793 & 95.25 & 94.31 & 7123.4 \\
\hline n100c & 18.0665 & 18.2033 & 95.19 & 94.47 & 7146.8 \\
\hline
\end{tabular}

Table VII Comparison between the MBS-OEA and 2 existing algorithms on floorplanning problems with soft rectangular blocks

\begin{tabular}{c||c|c||c|c||c|c||c|c||c|c}
\hline \multirow{2}{*}{} & \multicolumn{2}{c||}{ apte } & \multicolumn{2}{c||}{ xerox } & \multicolumn{2}{c||}{ hp } & \multicolumn{2}{c||}{ ami33 } & \multicolumn{2}{c|}{ ami49 } \\
\cline { 2 - 11 } & Area & $\begin{array}{c}\text { Time } \\
(\mathrm{s})\end{array}$ & Area & $\begin{array}{c}\text { Time } \\
(\mathrm{s})\end{array}$ & Area & $\begin{array}{c}\text { Time } \\
(\mathrm{s})\end{array}$ & Area & $\begin{array}{c}\text { Time } \\
(\mathrm{s})\end{array}$ & Area & $\begin{array}{c}\text { Time } \\
(\mathrm{s})\end{array}$ \\
\hline$[52]$ & 46.81 & 53.0 & 19.43 & 71.6 & 8.95 & 107.3 & 1.21 & 774.6 & 38.17 & 2354.0 \\
\hline CBL & 48.20 & 78 & 19.77 & 76 & 9.01 & 75 & 1.18 & 87 & 36.10 & 179 \\
\hline MBS-OEA & 46.56 & 18.5 & 19.35 & 21.2 & 8.83 & 26.1 & 1.16 & 361.4 & 35.77 & 890.3 \\
\hline
\end{tabular}

Table VIII Results of the MBS-OEA on floorplanning problems with hybrid blocks

\begin{tabular}{|c|c|c|c|c|c|}
\hline \multirow{2}{*}{ Problem name } & \multicolumn{2}{|c|}{ Area } & \multicolumn{2}{|c|}{ Area Ratio (\%) } & \multirow{2}{*}{$\begin{array}{c}\text { Average times } \\
\text { (s) }\end{array}$} \\
\hline & Best & Average & Best & Average & \\
\hline n030_10 & 24.0580 & 24.3365 & 98.34 & 97.22 & 478.2 \\
\hline n030_20 & 26.3923 & 26.6919 & 97.38 & 96.30 & 703.4 \\
\hline n050_10 & 24.4841 & 24.8773 & 97.91 & 96.37 & 1704.1 \\
\hline n050_20 & 26.9121 & 27.2971 & 96.26 & 94.91 & 2247.3 \\
\hline n100_10 & 19.5021 & 19.7964 & 94.23 & 92.83 & 9478.0 \\
\hline n100_20 & 21.4648 & 21.8053 & 92.79 & 91.34 & 12743.3 \\
\hline
\end{tabular}

Table IX compares the results of the MBS-OEA with those of website [48], and the running environment of [48] is Sun Sparc Ultra-II. As can be seen, the Areas of the MBS-OEA on 5 of the 6 problems are smaller than those of [48]. Only for n100_10, the Area of the MBS-OEA is slightly larger.

Table IX Comparison between the MBS-OEA and [48] on floorplanning problems with hybrid blocks

\begin{tabular}{|c|c|c|c|c|c|c|}
\hline & \multicolumn{2}{|c|}{ n030_10 } & \multicolumn{2}{|c|}{ n030_20 } & \multicolumn{2}{|c|}{ n050_10 } \\
\hline & Area & Time (s) & Area & Time (s) & Area & Time (s) \\
\hline [48] & 24.33 & 495 & 28.14 & 432 & 24.96 & 2001 \\
\hline \multirow[t]{3}{*}{ MBS-OEA } & 24.06 & 478 & 26.39 & 703 & 24.48 & 1704 \\
\hline & \multicolumn{2}{|c|}{ n050_20 } & \multicolumn{2}{|c|}{ n100_10 } & \multicolumn{2}{|c|}{ n100_20 } \\
\hline & Area & Time (s) & Area & Time (s) & Area & Time (s) \\
\hline [48] & 28.27 & 1807 & 19.30 & 16295 & 21.96 & 12697 \\
\hline MBS-OEA & 26.91 & 2247 & 19.50 & 9478 & 21.46 & 12743 \\
\hline
\end{tabular}




\section{Finding the Strength of the MBS-OEA}

Now that we have demonstrated the effectiveness of the MBS-OEA, it becomes necessary to identify the key component (or a combination of components) that is mainly responsible for the good performance. Because this paper first presents a new representation, the MBS, and then designs the OEA on the basis of the MBS, we should determine whether the MBS or the OEA contributes to the observed benefits. Thus, two groups of experiments are designed: the MBS and the OEA. The problems with 30 to 50 blocks in Subsection VI.A and $\mathrm{C}$ are used. The parameters are exactly the same as those of Section VI.

\section{A. The MBS}

Because the core of the MBS is to design four initial positions and move rules, the aim of this subsection is to examine whether all the four initial positions and move rules are mandatory. Two experiments are designed as follows: the first one uses only initial positions 2 and 3 and the corresponding move rules; whereas the second one uses only initial position 2 and the corresponding move rule. The first version of the MBS-OEA is labeled as MBS23-OEA and the second version as MBS2-OEA. The results are shown in Table X.

From the results shown in Table X, it is clear that the MBS23-OEA and the MBS2-OEA are outperformed by the MBS-OEA in both the best areas and the average areas for all the 12 problems. As indicated in Subsection II.C, the MBS can be considered an extension of the BL method. The results also illustrate that these extra choices for initial positions are useful and that the MBS is a successful development of the BL and the BLF methods.

Table X Comparison among the MBS-OEA, the MBS23-OEA, and the MBS2-OEA

\begin{tabular}{c|r|c|c|c|c|c}
\hline \multirow{2}{*}{$\begin{array}{c}\text { Problem } \\
\text { name }\end{array}$} & \multicolumn{3}{|c|}{ Best Area } & \multicolumn{3}{c}{ Average Area } \\
\cline { 2 - 7 } & MBS-OEA & MBS23-OEA & MBS2-OEA & MBS-OEA & MBS23-OEA & MBS2-OEA \\
\hline \hline ami33 & 1.175363 & 1.207605 & 1.200255 & 1.206013 & 1.217949 & 1.206924 \\
\hline
\end{tabular}




\begin{tabular}{l|l|l|l|l|l|l}
\hline ami49 & 36.350160 & 37.281552 & 36.728832 & 37.040805 & 37.525650 & 37.889630 \\
\hline n30a & 21.4600 & 21.6070 & 21.9224 & 21.7734 & 21.8891 & 22.1288 \\
\hline n30b & 20.1150 & 20.3643 & 21.7620 & 20.4226 & 20.6339 & 21.8907 \\
\hline n30c & 22.8242 & 23.0232 & 23.5520 & 23.2908 & 23.3169 & 23.8047 \\
\hline n50a & 20.4140 & 20.9084 & 21.0624 & 20.8561 & 21.2615 & 21.1870 \\
\hline n50b & 20.9271 & 21.5688 & 21.4464 & 21.2932 & 21.7856 & 21.6701 \\
\hline n50c & 20.7564 & 21.4650 & 20.9055 & 21.2032 & 21.7346 & 21.9233 \\
\hline n030_10 & 24.0580 & 24.1741 & 24.1577 & 24.3365 & 24.3790 & 24.5096 \\
\hline n030_20 & 26.3923 & 26.4476 & 26.7678 & 26.6919 & 26.8100 & 26.8294 \\
\hline n050_10 & 24.4841 & 24.7916 & 24.6288 & 24.8773 & 25.1249 & 25.7450 \\
\hline n050_20 & 26.9121 & 27.2682 & 27.7882 & 27.2971 & 27.4834 & 27.8437 \\
\hline
\end{tabular}

\section{B. The OEA}

The OEA can be viewed as a development of CGP-EAs (Subsection V.C), the main difference between the two is that CGP-EAs use fixed subpopulations whereas the OEA uses dynamic population. To test whether the dynamic population in the OEA is useful, an experiment is designed as follows: the splitting operator is deleted and each organization is randomly split into two organizations with the same size after performing the annexing operator. Thus, the size of each organization is always Max $O$. The corresponding version of the algorithm is labeled as MBS-FixOrg. The results are shown in Table XI.

On the other hand, the training operator in the OEA is equivalent to a heuristic search and can improve the members' qualities significantly. Thus, an experiment is designed to test whether the splitting and annexing operators are mandatory. In the experiment, the splitting and annexing operators are deleted and only the training operator is repeatedly performed on a randomly generated member, until the computational cost is the same as that of the MBS-OEA in Section VI. The corresponding version of the algorithm is labeled as the MBS-Training. The results are shown in Table XI.

Table XI Comparison among the MBS-OEA, the MBS-FixOrg, and the MBS-Training

\begin{tabular}{c|c|c|c|c|c|c}
\hline \multirow{2}{*}{$\begin{array}{c}\text { Problem } \\
\text { name }\end{array}$} & \multicolumn{3}{|c|}{ Best Area } & \multicolumn{3}{c}{ Average Area } \\
\cline { 2 - 7 } & MBS-OEA & MBS-FixOrg & MBS-Training & MBS-OEA & MBS-FixOrg & MBS-Training \\
\hline \hline
\end{tabular}




\begin{tabular}{l|l|l|l|l|l|l}
\hline \hline ami33 & 1.175363 & 1.195600 & 1.347108 & 1.206013 & 1.221301 & 1.414218 \\
\hline ami49 & 36.350160 & 36.959720 & 40.393640 & 37.040805 & 37.590801 & 42.053505 \\
\hline n30a & 21.4600 & 21.8356 & 23.0421 & 21.7734 & 22.0593 & 24.8564 \\
\hline n30b & 20.1150 & 20.3776 & 21.3110 & 20.4226 & 20.7287 & 22.6914 \\
\hline n30c & 22.8242 & 23.2312 & 24.3540 & 23.2908 & 23.4381 & 25.6381 \\
\hline n50a & 20.4140 & 20.8146 & 22.7493 & 20.8561 & 21.1277 & 23.9332 \\
\hline n50b & 20.9271 & 21.2833 & 22.9788 & 21.2932 & 21.6547 & 24.4680 \\
\hline n50c & 20.7564 & 21.2266 & 23.0580 & 21.2032 & 21.5862 & 24.0062 \\
\hline n030_10 & 24.0580 & 24.0727 & 25.4207 & 24.3365 & 24.3945 & 26.3575 \\
\hline n030_20 & 26.3923 & 27.3403 & 28.3898 & 26.6919 & 26.8514 & 29.3339 \\
\hline n050_10 & 24.4841 & 24.5768 & 26.6347 & 24.8773 & 24.9879 & 27.5649 \\
\hline n050_20 & 26.9121 & 27.0242 & 28.4485 & 27.2971 & 27.3845 & 30.4170 \\
\hline
\end{tabular}

From the results shown in Table XI, it is clear that the MBS-FixOrg and the MBS-Training are outperformed by the MBS-OEA in both the best areas and the average areas for all the 12 problems. Therefore, the results illustrate two points: (1) the dynamic population is better than the fixed subpopulations and (2) although the training operator can improve members' qualities significantly, it can be easily trapped in the local optima. The splitting and annexing operators are very useful. They not only maintain the dynamic population, but also prevent the solutions from being trapped in the local optima.

To conclude, the results of the above two subsections show that changing either the MBS or the OEA would worsen the performance. Thus, the good performance of the MBS-OEA benefits from both the MBS and the OEA.

\section{Conclusions and Future Works}

This paper first proposes a new representation for floorplanning, the MBS, and then proposes a new evolutionary algorithm, MBS-OEA, for solving floorplanning problems on the basis of the MBS. The MBS can directly handle both rectangular and rectilinear blocks and is suitable for designing effective crossover operators for EAs. Thus, the MBS is useful for boosting applications of EAs in the field of floorplanning. 
In the experiments, several kinds of problems are used to test the performance of the MBS-OEA. The number of blocks in these problems varies from 9 to 300. Moreover, the MBS-OEA is also compared with several well-designed existing algorithms. The results in Tables III-IX show that the MBS-OEA not only can find higher quality solutions for problems with both rectangular and rectilinear blocks, but also has the ability to solve large-scale problems. Additionally, all experimental results are obtained under the same parameter setting, which illustrates that the MBS-OEA has a stable performance and is easy to use.

Furthermore, to identify the key component responsible for the good performance of the MBS-OEA, experiments with limited initial positions in the MBS and modified versions of the OEA are performed. The results show that the good performance of the MBS-OEA benefits from both the MBS and the OEA.

In this paper, floorplanning is a general classical case. Because floorplanning is an essential step in the physical design of VLSI circuits, there are various modified versions like the one with preoccupied blocks. Some researchers even proposed new problem formulations opposed to the classical formulation. For example, [53] proposed a perfect rectilinear floorplanning formulation on the basis of the analyses of the shortcomings of the classical floorplanning formulation. Therefore, our future research will focus on extending the MBS and the MBS-OEA to handle the foregoing modified versions of floorplanning.

\section{Acknowledgment}

This work was supported by the National Natural Science Foundation of China under Grant 60502043, the Program for New Century Excellent Talents in University of China 
under Grant NCET-06-0857, and the Natural Science Research Project of Shaanxi, China.

\section{References}

1. H. Murata, K. Fujiyoshi, S. Nakatake, and Y. Kajitani, "VLSI module placement based on rectangle-packing by the sequence-pair,” IEEE Trans. CAD, 15(12), pp.1518-1524, 1996.

2. S. Nakatake, K. Fujiyoshi, H. Murata, and Y. Kajitani, "Module packing based on the BSG-structure and IC layout applications,” IEEE Trans. CAD, 17(6), pp.519-530, 1998.

3. J. M. Lin and Y. W. Chang, “TCG: a transitive closure graph-based representation for general floorplans,” IEEE Trans. VLSI, 13(2), pp.288-292, 2005.

4. J. M. Lin and Y. W. Chang, "TCG-S: orthogonal coupling of $\mathrm{P}^{*}$-admissible representations for general floorplans,' IEEE Trans. CAD, 23(6), pp.968-980, 2004.

5. J. M. Lin, Y. W. Chang, and S. P. Lin, "Corner sequence - a P-admissible floorplan representation with a worst case linear-time packing scheme,” IEEE Trans. VLSI, 11(4), pp.679-686, 2003.

6. L. Jiao, J. Jiu, and W. Zhong, “An organizational coevolutionary algorithm for classification,” IEEE Trans. Evol. Comput., 10(1), pp.67-80, 2006.

7. J. Liu, W. Zhong, and L. Jiao, “An organizational evolutionary algorithm for numerical optimization,” IEEE Trans. System, Man, and Cybernetics—Part B, 37(4), pp. 1052-1064, 2007.

8. J. Liu, W. Zhong, F. Liu, and L. Jiao, “An organizational evolutionary algorithm for SAT problem,” Chinese Journal of Computers, 27(10), pp.1422-1428, 2004. (in Chinese)

9. P. N. Guo, T. Takahashi, C. K. Cheng, and T. Yoshimura, "Floorplanning using a tree 
representation,” IEEE Trans. CAD, 20(2), pp.281-289, 2001.

10. Y. C. Chang, Y. W. Chang, G. M. Wu, and S. W. Wu, “ $B$-trees: a new representation for nonslicing floorplans,” in Proc. ACM/IEEE Design Automation Conf., pp.458-463, 2000.

11. X. Hong, S. Dong, G. Huang, Y. Cai, C. K. Cheng, and J. Gu, "Corner block list representation and its application to floorplan optimization,” IEEE Trans. Circuits and Systems-II: Express Briefs, 51(5), pp.228-233, 2004.

12. E. F. Y. Young, C. C. N. Chu, and Z. C. Shen, "Twin binary sequences: a nonredundant representation for general nonslicing floorplan,” IEEE Trans. CAD, 22(4), pp.457-469, 2003.

13. S. Y. Ho, S. J. Ho, Y. K. Lin, and W. C. C. Chu, “An orthogonal simulated annealing algorithm for large floorplanning problems,” IEEE Trans. VLSI, 12(8), pp.874-876, 2004.

14. W. Zhong, J. Liu, M. Xue, and L. Jiao, “A multiagent genetic algorithm for global numerical optimization,” IEEE Trans. Syst., Man, and Cybern. B, 34(2), pp.1128-1141, 2004.

15. J. Liu, W. Zhong, and L. Jiao, “A multiagent evolutionary algorithm for constraint satisfaction problems,” IEEE Trans. Syst., Man, and Cybern. B, 36(1), pp. 54-73, 2006.

16. C. Zhou, W. Xiao, T. M. Tirpak, and P. C. Nelson, "Evolving accurate and compact classification rules with gene expression programming,” IEEE Trans. Evol. Comput., 7(6), pp.519-531, 2003.

17. W. H. Au, K. C. C. Chan, and X. Yao, “A novel evolutionary data mining algorithm with applications to churn prediction,” IEEE Trans. Evol. Comput., 7(6), pp.532-545, 2003.

18. X. Yao and Y. Liu, “A new evolutionary system for evolving artificial neural networks,” 
IEEE Trans. Neural Networks, 8(3), pp.694-713, 1997.

19. J. P. Cohoon, S. U. Hedge, W. N. Martin, and D. S. Richards, "Distributed genetic algorithms for the floorplan design problem,” IEEE Trans. CAD, 10(4), pp.483-492, 1991.

20. V. Schnecke and O. Vornberger, "Hybrid genetic algorithms for constrained placement problems,” IEEE Trans. Evol. Comput., 1(4), pp.266-277, 1997.

21. C. L. Valenzuela and P. Y. Wang, "VLSI placement and area optimization using a genetic algorithm to breed normalized postfix expressions,” IEEE Trans. Evol. Comput., 6(4), pp.390-401, 2002.

22. J. Xu, P. N. Guo, and C. K. Cheng, "Rectilinear block placement using sequence-pair," in Proc. ISPD, pp.173-178, 1998.

23. M. Z. Kang and W. W. M. Dai, “Arbitrary rectilinear block packing based on sequence pair,” in Proc. ICCAD, pp.259-266, 1998.

24. K. Fujiyoshi and H. Murata, “Arbitrary convex and concave rectilinear block packing using sequence-pair,” IEEE Trans. CAD, 19(2), pp.224-233, 2000.

25. S. Nakatake, M. Furuya, and Y. Kajitani, "Module placement on BSG-structure with pre-placed modules and rectilinear modules,” in Proc. ASP-DAC, pp.571-576, 1998.

26. M. Z. Kang and W. W. M. Dai, “Topology constrained rectilinear block packing for layout reuse,” in Proc. ISPD, pp.179-186, 1998.

27. Y. Pang, C. K. Cheng, K. Lampasert, and W. Xie, "Rectilinear block packing using O-tree representation,” in Proc. ISPD, pp.156-161, 2001.

28. G. M. Wu, Y. C. Chang, and Y. W. Chang, "Rectilinear block placement using $B^{*}$-trees," 
in Proc. ICCD, pp.351-356, 2000.

29. Y. Ma, X. Hong, S. Dong, Y. Cai, C. K. Cheng, and J. Gu, "Floorplanning with abutment constraints and L-shaped/T-shaped blocks based on corner block list,” in Proc. DAC, pp.770-775, 2001.

30. J. M. Lin, H. L. Chen, and Y. W. Chang, “Arbitrarily shaped rectilinear module placement using the transitive closure graph representation,” IEEE Trans. VLSI, 10(6), pp.886-901, 2002.

31. C. C. N. Chu and E. F. Y. Young, “ Nonrectangular shaping and sizing of soft modules for floorplan-design improvement,” IEEE Trans. CAD, 23(1), pp.71-79, 2004.

32. B. S. Baker, E. G. Coffman, Jr., and R. L. Rivest, "Orthogonal packings in two dimensions,” SIAM J. Comput., 9(4), pp. 808-826, 1980.

33. B. Chazelle, “The bottom-left bin packing heuristic: An efficient implementation,” IEEE Trans. Comput., 32(8), pp. 697-707, 1983.

34. E. K. Burke, G. Kendall, and G. Whitwell, "A new placement heuristic for the orthogonal stock-cutting problem,” Operations Research, 52(4), pp. 655-671, 2004.

35. E. K. Burke, R. S. R. Hellier, G. Kendall, and G. Whitwell, "A new bottom-left-fill heuristic algorithm for the two-dimensional irregular packing problem,” Operations Research, 54(3), pp. 587-601, 2006.

36. D. Smith, "Bin-packing with adaptive search," in Proc. of the Int. Conf. on Genetic Algorithms and their Applications, Lawrence Erlbaum, pp. 202-206, 1985.

37. L. Davis, “Applying adaptive search algorithms to epistatic domains,” in Proc. of the $9^{\text {th }}$ Int. Joint Conf. on Artificial Intelligence, Los Angeles, pp. 162-164, 1985. 
38. P. Prosser, “A hybrid genetic algorithm for pallet loading," in Proc. of the 8th European Conference on Artificial Intelligence, Pitman, London, pp. 159-164, 1988.

39. D. Liu and H. Teng, “An improved BL-algorithm for genetic algorithms of the orthogonal packing of rectangles.” European Journal of Operational Research, 112, pp. 413-419, 1999.

40. K. A. Dowsland, E. A. Herbert, G. Kendall, and E. Burke, "Using tree search bounds to enhance a genetic algorithm approach to two rectangle packing problems,” European Journal of Operational Research, 168, pp. 390-402, 2006.

41. E. Hopper and B. Turton, "Application of genetic algorithms to packing problems - a review," in Proc. of the $2^{\text {nd }}$ On-line World Conference on Soft Computing in Engineering Design and Manufacturing, Springer Verlag, London, pp. 279-288, 1997.

42. E. Hopper and B. C. H. Turton, "An empirical investigation of meta-heuristic and heuristic algorithms for a 2D packing problem,” European Journal of Operational Research, 128, pp. 34-57, 2001.

43. R. H. Coase, The Firm, the Market, and the Law, Chicago: University of Chicago Press, 1988.

44. J. R. Wilcox, Organizational Learning within a Learning Classifier System, Master thesis, University of Illinois, 1995. Also Tech. Report No. 95003 IlliGAL.

45. J. P. Cohoon, S. U. Hegde, W. N. Martin, and D. S. Richards, "Punctuated equilibria: a parallel genetic algorithm,” in Proc. of $2^{\text {nd }}$ Int. Conf. on Genetic Algorithms, Cambridge, MA, USA, pp.148-154, 1987.

46. B. Kroger, P. Schwenderling, and O. Vornberger, "Parallel genetic packing of rectangles," 
in Proc. of the $1^{\text {st }}$ Int. Work Shop on Parallel Problem Solving from Nature (PPSN-90), pp. 8-11, 1990.

47. B. B. Prahlada and R. C. Hansdah, "Extended distributed genetic algorithm for channel routing," in Proc. of the 5th IEEE Symposium on Parallel and Distributed Processing, pp. 726-733, 1993.

48. J. Rabaey, Gigascale systems research center, [Online] Available: http://www.cse.ucsc.edu/research/surf/GSRC/progress.html, last accessed Aug. 2005.

49. Y. Pang, C. K. Cheng, and T. Yoshimura, “An enhanced perturbing algorithm for floorplan desigh using the O-tree representation,” in Proc. ACM Int. Physical Design Symposia, pp.168-173, 2000.

50. X. Tang and D. F. Wong, "FAST-SP: a fast algorithm for block placement based on sequence pair,” in Proc. IEEE Asia South Pacific Design Automation Conf., pp.521-526, 2001.

51. C. Zhuang, K. Sakanushi, L. Jin, and Y. Kajitani, “An enhanced Q-sequence augmented with empty-room-insertion and parenthesis trees," in Proc. Design Automation Test Europe, pp.61-68, 2002.

52. F. Y. Young, C. C. N. Chu, W. S. Luk, and Y. C. Wong, "Handling soft modules in general nonslicing floorplan using lagrangian relaxation,” IEEE Trans. CAD, 20(5), pp. 687-692, 2001.

53. A. B. Kahng, “Classical floorplanning harmful?,” in Proc. of ISPD 2000, San Diego, CA, pp. 207-213, 2000 